\title{
Vibration and acoustic response of rectangular sandwich plate under thermal environment
}

\author{
Yuan Liu and Yueming $\mathrm{Li}^{*}$ \\ State Key Laboratory for Strength and Vibration of Mechanical Structures, Xi'an Jiaotong University, Xi'an, \\ Shaanxi, China
}

Received 15 October 2012

Revised 20 March 2013

Accepted 12 May 2013

\begin{abstract}
In this paper, we focus on the vibration and acoustic response of a rectangular sandwich plate which is subjected to a concentrated harmonic force under thermal environment. The critical buckling temperature is obtained to decide the thermal load. The natural frequencies and modes as well as dynamic responses are acquired by using the analytical formulations based on equivalent non-classical theory, in which the effects of shear deformation and rotational inertia are taken into account. The rise of thermal load decreases the natural frequencies and moves response peaks to the low-frequency range. The specific features of sandwich plates with different formations are discussed subsequently. As the thickness ratio of facing to core increases, the natural frequencies are enlarged, and the response peaks float to the high-frequency region. Raising the Young's modulus of the core can cause the similar trends. The accuracy of the theoretical method is verified by comparing its results with those computed by the FEM/BEM.
\end{abstract}

Keywords: Vibration, acoustic response, thermal environment, sandwich plate

\section{Nomenclature}

$\begin{array}{llll}a, b & \text { Length and width of the plate } & T_{o} & \text { Reference temperature } \\ h, h_{1}, h_{2} & \text { Thicknesses } & T & \text { Working temperature } \\ E_{1}, E_{2} & \text { Young's moduli } & \Delta T & \text { Temperature difference } \\ \nu_{1}, \nu_{2} & \text { Poisson's ratios } & t & \text { Time } \\ \rho_{1}, \rho_{2} & \text { Densities } & \omega_{m n}^{(p)} & \text { Natural frequency } \\ \alpha_{1}, \alpha_{2} & \text { Thermal expansion coefficients } & T_{m n}^{(p)}(t) & \text { Time function } \\ G_{z} & \text { Equivalent shear modulus } & P_{m n}^{(p)}(t) & \text { Generalized force } \\ k_{\tau} & \text { Shear deformation coefficient } & M_{m n}^{(p)}(t) & \text { Generalized mass } \\ w & \text { Transverse deflection } & \omega & \text { Excitation frequency } \\ \beta_{x}, \beta_{y} & \text { Generalized rotations } & q_{0} & \text { Amplitude of the load } \\ \rho & \text { Equivalent density } & c & \text { Sound velocity } \\ N_{x}, N_{y}, N_{x y} & \text { Membrane forces } & \eta & \text { Structural damping ratio } \\ q & \text { Concentrated force } & Q_{x}, Q_{y} & \text { Transverse shear force } \\ D & \text { Equivalent flexural rigidity } & M_{x}, M_{y} & \text { Bending moments }\end{array}$

* Corresponding author: Yueming Li, State Key Laboratory for Strength and Vibration of Mechanical Structures, Xi' an Jiaotong University, Xi'an 710049, Shaanxi, China. Tel.: +86 0298266 8340; Fax: +86 0298266 9044; E-mail: liyueming@mail.xjtu.edu.cn. 
$\nu \quad$ Equivalent poisson's ratio $\quad M_{x y} \quad$ Twisting moment

$J \quad$ Equivalent rotational inertia

\section{Introduction}

Sandwich plates are extensively used in the aerospace industry due to their high ratios of strength and stiffness to weight and other good qualities, such as heat resistant and sound insulation. As common components of the thermal protection system, sandwich plates are exposed to high temperature caused by aerodynamic heating in their service environment. Thermal environment could influence the vibration and acoustic characteristics of the sandwich plate. In order to use them efficiently, it is needed to have a good understanding of intrinsic property and an accurate prediction of vibration and sound radiation characteristics of sandwich plates under thermal environment. Sandwich plates with different formations show different features, thus the analyses of natural and dynamic characteristics of various sandwich plates contribute to getting thorough knowledge about sandwich plates.

As a typical type of composite plates, the sandwich plate is studied by using two major analytical methods. One is sub-microscopic theory, in which each layer of the plate is regarded as a mechanical unit. The deformation and equilibrium of each unit and their compatibility between units are considered in this theory, and the structural analysis or finite element method (FEM) is used to solve the mechanical problem of sandwich plates. Various studies on sandwich plates by using the sub-microscopic theory are present in different research fields. Tu [1] has developed non-linear differential equations for the calculation of the deflections and buckling loads of sandwich plates subjected to transverse loads and edgewise shears and compressions. Qin et al. [2] have investigated the low velocity impact response of a corrugated core sandwich plate. Xin and Lu [3] have developed a theoretical model for sound radiation of an orthogonally rib-stiffened sandwich structure.

The sub-microscopic theory, which takes the inherent morphology and arrangement of layers into account, appears to be very efficient in the analysis of sandwich plates. However, the governing equations established by this method are simultaneous equations with numerous variables, thus the analytical solutions cannot be obtained in general. The macroscopic equivalent theory, which transforms the sandwich plates to equivalent homogeneous anisotropic plates, is developed to get the analytical solutions of sandwich plates. In this method, the structural and material properties of the sandwich plates are presented in equivalent compositional parameters, and the mechanical behaviors are studied by using traditional anisotropy theories. Romanoff and Varsta [4] have presented a theory for the bending response of web-core sandwich plates, in which a discrete core is transformed into an equivalent homogenous continuum. Based on the non-classical theory for medium and thick plate, Tsao [5] has proposed a method, namely equivalent non-classical theory, to analyze the dynamic response of sandwich plates by considering the effects of shear deformation, rotational inertia and compressional deformation. By comparing with other analytical and numerical methods, the equivalent non-classical theory is demonstrated to be concise and efficient in studying the sandwich plates.

Sound radiation of vibrating sandwich plates has been studied in recent years. Omrani and Tawfiq [6] have carried out a vibro-acoustic analysis of a micro-perforated sandwich structure and developed a numerical local impedance model to describe the influence induced by the perforation through software. Assaf et al. [7] have given a finite element formulation, which takes the effects of fluid loading into consideration, to conduct the vibro-acoustic analysis of damped sandwich plates. Sorokin [8] has done a vibro-acoustic analysis of a submerged sandwich plate, in which a two-dimensional formulation relevant to cylindrical bending of a plate is explored. Foin and colleagues [9] have analyzed the vibro-acoustic problem of sandwich plates submerged in fluid theoretically.

Although a number of analyses have been presented for vibro-acoustic problems of sandwich plates, few investigations can be found for them under thermal environment, especially, none for theoretical investigations. Jeyaraj et al. [10] have presented studies on vibration and sound radiation characteristics of a composite plate under thermal environment numerically. The inherent material damping property is considered in their studies. The buckling temperature and vibration responses are acquired by FEM, while the sound radiation is calculated by a coupled FEM/BEM (boundary element method) technique. Kumar et al. [11] have carried out studies on vibration and acoustic responses of various orthotropic composite circular discs under thermal environment. In their study, commercial software ANSYS is used to obtain the vibration data which are input to another commercial software LMS SYSNOISE to calculate the acoustic response. 


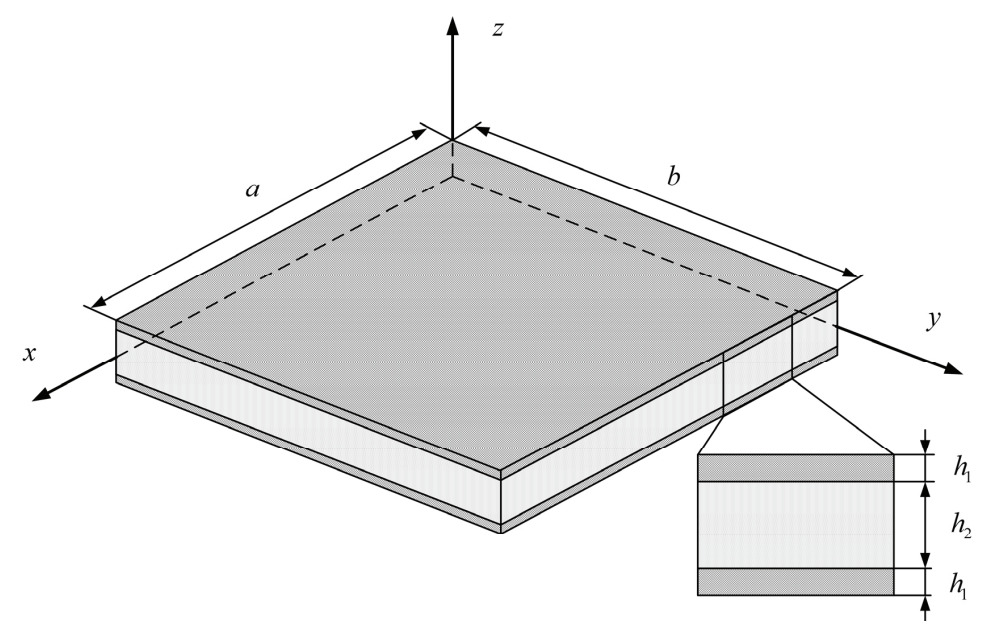

Fig. 1. Sandwich plate model.

Li et al. $[12,13,14]$ have carried out vibro-acoustic analyses for structures under thermal environment, including the broadband vibro-acoustic response based on hybrid FE-SEA (Finite Element-Statistical Energy Analysis) [12], the influence of thermal stress with numerical method [13], and analytical solution for the dynamic and acoustic characteristics of an isotropic plate under thermal environments using classical plate theory [14].

The aim of this paper is to analyze the vibration and acoustic characteristics of a sandwich plate subjected to a concentrated harmonic force under thermal environment. The analytical method based on the equivalent nonclassical theory is developed and numerical simulation is performed to verify the accuracy of this method. The influences caused by thermal environment on the sandwich and the specific features of various sandwich plates are deeply discussed in the present study.

\section{Fundamental formulations}

Here we consider a sandwich plate with dimensions of $a \times b \times\left(2 h_{1}+h_{2}\right)$, in which the thickness for top and bottom facings is $h_{1}$ and the thickness for core is $h_{2}$, as shown in Fig. 1. Thereinafter, the variables with subscript 1 are defined for the facings while those with subscript 2 for the core. The plate consists of two isotropic materials for the facings and core respectively. The facings are made of the material of $E_{1}, \nu_{1}, \rho_{1}, \alpha_{1}$ and the core is made of the material of $E_{2}, \nu_{2}, \rho_{2}, \alpha_{2}$. The middle surface of sandwich plate is lying on the $x-y$ plane.

\subsection{Governing equations}

Based on the non-classical theory for medium and thick plates, and by adopting the Власов еquations, the governing equations for dynamic analysis considering the membrane forces are derived as Eq. (1) (Detailed mathematical derivation of these equations can be found in the Appendix):

$$
\left\{\begin{array}{l}
\frac{G_{z} h}{k_{\tau}}\left(\nabla^{2} w+\frac{\partial \beta_{x}}{\partial x}+\frac{\partial \beta_{y}}{\partial y}\right)-\rho h \frac{\partial^{2} w}{\partial t^{2}}+N_{x} \frac{\partial^{2} w}{\partial x^{2}}+N_{y} \frac{\partial^{2} w}{\partial y^{2}}+2 N_{x y} \frac{\partial^{2} w}{\partial x \partial y}+q=0 \\
D\left(\frac{\partial^{2} \beta_{x}}{\partial x^{2}}+\frac{1-\nu}{2} \frac{\partial^{2} \beta_{x}}{\partial y^{2}}+\frac{1+\nu}{2} \frac{\partial^{2} \beta_{y}}{\partial x \partial y}\right)-\frac{G_{z} h}{k_{\tau}}\left(\beta_{x}+\frac{\partial w}{\partial x}\right)-\rho J \frac{\partial^{2} \beta_{x}}{\partial t^{2}}=0 \\
D\left(\frac{\partial^{2} \beta_{y}}{\partial y^{2}}+\frac{1-\nu}{2} \frac{\partial^{2} \beta_{y}}{\partial x^{2}}+\frac{1+\nu}{2} \frac{\partial^{2} \beta_{x}}{\partial x \partial y}\right)-\frac{G_{z} h}{k_{\tau}}\left(\beta_{y}+\frac{\partial w}{\partial y}\right)-\rho J \frac{\partial^{2} \beta_{y}}{\partial t^{2}}=0
\end{array}\right.
$$


where $h=2 h_{1}+h_{2} . N_{x}, N_{y}$ and $N_{x y}$ are membrane forces, for this paper, they are generated by thermal stresses. $G_{z}, D, \nu, \rho h$ and $\rho J$ are equivalent compositional parameters defined as Eqs (2) to (6):

$$
\begin{aligned}
G_{z} & =\left[1-3\left(\frac{h_{2}}{2 h}-\frac{h_{2}^{3}}{6 h^{3}}\right)\right] \frac{E_{1}}{2\left(1+\nu_{1}\right)}+3\left(\frac{h_{2}}{2 h}-\frac{h_{2}^{3}}{6 h^{3}}\right) \frac{E_{2}}{2\left(1+\nu_{2}\right)} \\
D & =\frac{2 E_{1}}{3\left(1-\nu_{1}^{2}\right)}\left[\left(\frac{h}{2}\right)^{3}-\left(\frac{h_{2}}{2}\right)^{3}\right]+\frac{E_{2}}{3\left(1-\nu_{2}^{2}\right)}\left[\left(\frac{h_{2}}{2}\right)^{3}-\left(-\frac{h_{2}}{2}\right)^{3}\right] \\
\nu & =\frac{1}{3 D}\left\{\frac{2 E_{1} \nu_{1}}{1-\nu_{1}^{2}}\left[\left(\frac{h}{2}\right)^{3}-\left(\frac{h_{2}}{2}\right)^{3}\right]+\frac{E_{2} \nu_{2}}{1-\nu_{2}^{2}}\left[\left(\frac{h_{2}}{2}\right)^{3}-\left(-\frac{h_{2}}{2}\right)^{3}\right]\right\} \\
\rho h & =2 \rho_{1} h_{1}+\rho_{2} h_{2} \\
\rho J & =\frac{2}{3} \rho_{1}\left[\left(\frac{h}{2}\right)^{3}-\left(\frac{h_{2}}{2}\right)^{3}\right]+\frac{1}{3} \rho_{2}\left[\left(\frac{h_{2}}{2}\right)^{3}-\left(-\frac{h_{2}}{2}\right)^{3}\right]
\end{aligned}
$$

Assume the plate is stress-free at the reference temperature $T_{0}$. If the plate works under the steady temperature $T$, which is different from $T_{0}$, the stress state of the plate will be changed by the thermal stresses. In this study, simply supported boundary conditions along the edges are considered and the temperature is assumed to be uniformly distributed, so the thermal stresses caused by the temperature can be expressed as Eq. (7) [15]:

$$
\sigma_{x}=-\frac{E_{i}}{1-\nu_{i}} \alpha_{i} \Delta T \quad \sigma_{y}=-\frac{E_{i}}{1-\nu_{i}} \alpha_{i} \Delta T \quad \sigma_{x y}=0 \quad(i=1 \text { or } 2)
$$

where $\Delta T=T-T_{0}$. The membrane forces induced by the thermal stresses can be obtained by integrating Eq. (7) along the thickness, expressed as Eq. (8):

$$
\left\{\begin{array}{l}
N_{x} \\
N_{y} \\
N_{x y}
\end{array}\right\}=\int_{-\frac{2 h_{1}+h_{2}}{2}}^{\frac{2 h_{1}+h_{2}}{2}}\left\{\begin{array}{l}
\sigma_{x} \\
\sigma_{y} \\
\sigma_{x y}
\end{array}\right\} \mathrm{d} z
$$

Substituting Eq. (7) into Eq. (8), the membrane forces for the sandwich plate shown in Fig. 1 can be acquired as Eq. (9):

$$
N_{x}=\sum_{k=1}^{3} \frac{-E_{k} \alpha_{k} h_{k} \Delta T}{1-\nu_{k}} \quad N_{y}=\sum_{k=1}^{3} \frac{-E_{k} \alpha_{k} h_{k} \Delta T}{1-\nu_{k}} \quad N_{x y}=0
$$

\subsection{Natural frequencies and modes}

Considering the boundary conditions, we assume the mode shapes $W_{m n}^{(p)}, \Psi_{x m n}^{(p)}, \Psi_{y m n}^{(p)}$ as Eq. (10):

$$
\left\{\begin{array}{l}
W_{m n}^{(p)}=A_{m n}^{(p)} \sin \frac{m \pi}{a} x \sin \frac{n \pi}{b} y \\
\Psi_{x m n}^{(p)}=B_{m n}^{(p)} \cos \frac{m \pi}{a} x \sin \frac{n \pi}{b} y \\
\Psi_{y m n}^{(p)}=C_{m n}^{(p)} \sin \frac{m \pi}{a} x \cos \frac{n \pi}{b} y
\end{array}\right.
$$

where the $A_{m n}^{(p)}, B_{m n}^{(p)}, C_{m n}^{(p)}$ are mode coefficients and $m, n, p$ are indicators to specify mode shapes.

Suppose the displacement responses can be expressed as Eq. (11):

$$
w=W_{m n}^{(p)} \mathrm{e}^{\mathrm{i} \omega_{m n}^{(p)} t} \quad \beta_{x}=\Psi_{x m n}^{(p)} \mathrm{e}^{\mathrm{i} \omega_{m n}^{(p)} t} \quad \beta_{y}=\Psi_{y m n}^{(p)} \mathrm{e}^{\mathrm{i} \omega_{m n}^{(p)} t}
$$


When substitute Eq. (11) into Eq. (1), and let $q$ to be zero, we can obtain Eq. (12):

$$
\left\{\begin{array}{l}
\frac{G_{z} h}{k_{\tau}}\left(\nabla^{2} W_{m n}^{(p)}+\frac{\partial \Psi_{x m n}^{(p)}}{\partial x}+\frac{\partial \Psi_{y m n}^{(p)}}{\partial y}\right)+\rho h \omega_{m n}^{(p)^{2}} W_{m n}^{(p)}+N_{x} \frac{\partial^{2} W_{m n}^{(p)}}{\partial x^{2}}+N_{y} \frac{\partial^{2} W_{m n}^{(p)}}{\partial y^{2}}+2 N_{x y} \frac{\partial^{2} W_{m n}^{(p)}}{\partial x \partial y}=0 \\
D\left(\frac{\partial^{2} \Psi_{x m n}^{(p)}}{\partial x^{2}}+\frac{1-\nu}{2} \frac{\partial^{2} \Psi_{x m n}^{(p)}}{\partial y^{2}}+\frac{1+\nu}{2} \frac{\partial^{2} \Psi_{y m n}^{(p)}}{\partial x \partial y}\right)-\frac{G_{z} h}{k_{\tau}}\left(\Psi_{x m n}^{(p)}+\frac{\partial W_{m n}^{(p)}}{\partial x}\right)+\rho J \omega_{m n}^{(p)^{2}} \Psi_{x m n}^{(p)}=0 \\
D\left(\frac{\partial^{2} \Psi_{y m n}^{(p)}}{\partial y^{2}}+\frac{1-\nu}{2} \frac{\partial^{2} \Psi_{y m n}^{(p)}}{\partial x^{2}}+\frac{1+\nu}{2} \frac{\partial^{2} \Psi_{x m n}^{(p)}}{\partial x \partial y}\right)-\frac{G_{z} h}{k_{\tau}}\left(\Psi_{y m n}^{(p)}+\frac{\partial W_{m n}^{(p)}}{\partial y}\right)+\rho J \omega_{m n}^{(p)^{2}} \Psi_{y m n}^{(p)}=0
\end{array}\right.
$$

Substituting Eq. (10) into Eq. (12), one can get a homogeneous linear equations set about $A_{m n}^{(p)}, B_{m n}^{(p)}$ and $C_{m n}^{(p)}$. According to the mathematics, if there are nonzero solutions for homogeneous linear equations set, the number of solutions should be infinite. So we set $A_{m n}^{(p)}$ to be 1 , and let $b_{m n}^{(p)}, c_{m n}^{(p)}$ take the place of $B_{m n}^{(p)}$ and $C_{m n}^{(p)}$ respectively, where $b_{m n}^{(p)}=B_{m n}^{(p)} / A_{m n}^{(p)}$ and $c_{m n}^{(p)}=C_{m n}^{(p)} / A_{m n}^{(p)}$. Thus mode shapes can be rewritten as Eq. (13):

$$
\left\{\begin{array}{l}
W_{m n}^{(p)}=\sin \frac{m \pi}{a} x \sin \frac{n \pi}{b} y \\
\bar{\Psi}_{x m n}^{(p)}=b_{m n}^{(p)} \cos \frac{m \pi}{a} x \sin \frac{n \pi}{b} y \\
\bar{\Psi}_{y m n}^{(p)}=c_{m n}^{(p)} \sin \frac{m \pi}{a} x \cos \frac{n \pi}{b} y
\end{array}\right.
$$

The necessary and sufficient condition of homogeneous linear equations set existing nonzero solutions is that its determinant of the coefficient matrix is zero. So the natural frequency $\omega_{m n}^{(p)}$ can be acquired by solving Eq. (14):

$$
\left|\begin{array}{lll}
\frac{G_{z} h}{k_{\tau}}\left(-\frac{m^{2} \pi^{2}}{a^{2}}-\frac{n^{2} \pi^{2}}{b^{2}}\right)+\omega_{m n}^{(p)^{2}} \rho h-\frac{m^{2} \pi^{2}}{a^{2}} N_{x}-\frac{n^{2} \pi^{2}}{b^{2}} N_{y} & -\frac{G_{z} h}{k_{\tau}} \frac{m \pi}{a} & -\frac{G_{z} h}{k_{\tau}} \frac{n \pi}{b} \\
-\frac{G_{z} h}{k_{\tau}} \frac{m \pi}{a} & D\left(-\frac{m^{2} \pi^{2}}{a^{2}}-\frac{1-\nu}{2} \frac{n^{2} \pi^{2}}{b^{2}}\right)-\frac{G_{z} h}{k_{\tau}}+\rho J \omega_{m n}^{(p)^{2}} & -\frac{1+\nu}{2} D \frac{m n \pi^{2}}{a b} \\
-\frac{G_{z} h}{k_{\tau}} \frac{n \pi}{b} & -\frac{1+\nu}{2} D \frac{m n \pi^{2}}{a b} & D\left(-\frac{n^{2} \pi^{2}}{b^{2}}-\frac{1-\nu}{2} \frac{m^{2} \pi^{2}}{a^{2}}\right)-\frac{G_{z} h}{k_{\tau}}+\rho J \omega_{m n}^{(p)^{2}}
\end{array}\right|=0
$$

While $\omega_{m n}^{(p)}=0$, the critical buckling temperature can be obtained by Eq. (14).

When the temperature load is given, three positive frequencies corresponding to low-frequency, mediumfrequency and high-frequency can be solved. The low-frequency is the one relative to the result got by the classical plate theory. By putting the natural frequencies into the equations, the coefficients can be expressed as Eqs (15) to (16):

$$
\begin{aligned}
& b_{m n}^{(p)}=\frac{\frac{G_{z} h}{k_{\tau}}\left(-\frac{m^{2} \pi^{2}}{a^{2}}-\frac{n^{2} \pi^{2}}{b^{2}}\right)+\omega_{m n}^{(p) 2} \rho h-\frac{m^{2} \pi^{2}}{a^{2}} N_{x}-\frac{n^{2} \pi^{2}}{b^{2}} N_{y}}{\frac{G_{z} h}{k_{\tau}}\left(\frac{m \pi}{a}+\frac{n \pi}{b} \frac{D\left(-\frac{m \pi}{a}-\frac{1-\nu}{2} \frac{a n^{2} \pi}{b^{2} m}\right)-\frac{G_{z} h a}{k_{\tau} m \pi}+\frac{\rho J \omega_{m n}^{(p) 2} a}{m \pi}+\frac{1+\nu}{2} D \frac{m \pi}{a}}{D\left(-\frac{n \pi}{b}-\frac{1-\nu}{2} \frac{b m^{2} \pi}{a^{2} n}\right)-\frac{G_{z} h b}{k_{\tau} n \pi}+\frac{\rho J \omega_{m n}^{(p) 2} b}{n \pi}+\frac{1+\nu}{2} D \frac{n \pi}{b}}\right)} \\
& c_{m n}^{(p)}=\frac{\frac{G_{z} h}{k_{\tau}}\left(-\frac{m^{2} \pi^{2}}{a^{2}}-\frac{n^{2} \pi^{2}}{b^{2}}\right)+\omega_{m n}^{(p) 2} \rho h-\frac{m^{2} \pi^{2}}{a^{2}} N_{x}-\frac{n^{2} \pi^{2}}{b^{2}} N_{y}}{\frac{G_{z} h}{k_{\tau}}\left(\frac{m \pi}{a} \frac{D\left(-\frac{n \pi}{b}-\frac{1-\nu}{2} \frac{b m^{2} \pi}{a^{2} n}\right)-\frac{G_{z} h b}{k_{\tau} n \pi}+\frac{\rho J \omega_{m n}^{(p) 2} b}{n \pi}+\frac{1+\nu}{2} D \frac{n \pi}{b}}{D\left(-\frac{m \pi}{a}-\frac{1-\nu}{2} \frac{a n^{2} \pi}{b^{2} m}\right)-\frac{G_{z} h a}{k_{\tau} m \pi}+\frac{\rho J \omega_{m n}^{(p) 2} a}{m \pi}+\frac{1+\nu}{2} D \frac{m \pi}{a}}\right)}
\end{aligned}
$$


Considering Eqs (13), (15) and (16), we can determine the mode shapes.

\subsection{Dynamic analysis}

Let us analyze forced vibration of the sandwich plate which is subjected to a concentrated harmonic force. On the basis of mode superposition principle, the dynamic displacement responses can be expanded as Eq. (17):

$$
\left\{\begin{array}{l}
w(x, y, t)=\sum_{m} \sum_{n} \sum_{p} W_{m n}^{(p)}(x, y) T_{m n}^{(p)}(t) \\
\beta_{x}(x, y, t)=\sum_{m} \sum_{n} \sum_{p} \Psi_{x m n}^{(p)}(x, y) T_{m n}^{(p)}(t) \\
\beta_{y}(x, y, t)=\sum_{m} \sum_{n} \sum_{p} \Psi_{y m n}^{(p)}(x, y) T_{m n}^{(p)}(t)
\end{array}\right.
$$

Since $W_{m n}^{(p)}, \Psi_{x m n}^{(p)}$ and $\Psi_{y m n}^{(p)}$ have been obtained above, the next step is to get the expression of $T_{m n}^{(p)}(t)$.

Substituting Eq. (17) into Eq. (1), in view of Eq. (12), one can acquire Eqs (18) to (20):

$$
\begin{aligned}
& \sum_{m, n, p}\left[\ddot{T}_{m n}^{(p)}(t)+\omega_{m n}^{(p) 2} T_{m n}^{(p)}(t)\right] \rho h W_{m n}^{(p)}-q(x, y, t)=0 \\
& \sum_{m, n, p}\left[\ddot{T}_{m n}^{(p)}(t)+\omega_{m n}^{(p) 2} T_{m n}^{(p)}(t)\right] \rho J \Psi_{x m n}^{(p)}=0 \\
& \sum_{m, n, p}\left[\ddot{T}_{m n}^{(p)}(t)+\omega_{m n}^{(p) 2} T_{m n}^{(p)}(t)\right] \rho J \Psi_{y m n}^{(p)}=0
\end{aligned}
$$

The orthogonality for the mode shapes of the sandwich plate is given by Eq. (21):

$$
\iint\left[\rho h W_{m n}^{(p)} W_{k l}^{(q)}+\rho J\left(\Psi_{x m n}^{(p)} \Psi_{x k l}^{(q)}+\Psi_{y m n}^{(p)} \Psi_{y k l}^{(q)}\right)\right] \mathrm{d} x \mathrm{~d} y \begin{cases}=0 & m \neq k \text { or } n \neq l \text { or } p \neq q \\ \neq 0 & m=k \text { and } n=l \text { and } p=q\end{cases}
$$

Multiply Eqs (18), (19) and (20) by $W_{k l}^{(q)}, \Psi_{x k l}^{(q)}$ and $\Psi_{y k l}^{(q)}$ respectively, then add them together and integrate on the plate surface. By using the orthogonality for the modes, Eq. (22) with respect to $T_{m n}^{(p)}(t)$ could be obtained:

$$
\ddot{T}_{m n}^{(p)}(t)+\omega_{m n}^{(p)^{2}} T_{m n}^{(p)}(t)=\frac{P_{m n}^{(p)}(t)}{M_{m n}^{(p)}}
$$

in which generalized force and generalized mass can be defined as Eqs (23) and (24):

$$
\begin{aligned}
P_{m n}^{(p)}(t) & =\iint q(x, y, t) W_{m n}^{(p)}(x, y) \mathrm{d} x \mathrm{~d} y \\
M_{m n}^{(p)} & =\iint\left[\rho h W_{m n}^{(p)^{2}}+\rho J\left(\Psi_{x m n}^{(p)^{2}}+\Psi_{y m n}^{(p)^{2}}\right)\right] \mathrm{d} x \mathrm{~d} y
\end{aligned}
$$

The force $q$ is considered to be a concentrated harmonic force with frequency $\omega$ imposed on the point $\left(x_{0}, y_{0}\right)$, along $z$-axis, which can be expressed as Eq. (25):

$$
q(x, y, t)=q_{0} \delta\left(x-x_{0}, y-y_{0}\right) \mathrm{e}^{\mathrm{i} \omega t}
$$

where $q_{0}$ is the amplitude of the load.

In view of Eq. (25), one can obtain Eq. (26):

$$
T_{m n}^{(p)}(t)=\frac{q_{0} \sin \frac{m \pi x_{0}}{a} \sin \frac{n \pi y_{0}}{b} \mathrm{e}^{\mathrm{i} \omega t}}{\left(\omega_{m n}^{(p) 2}-\omega^{2}\right)\left\{\frac{a b}{4} \times\left[\rho h+\rho J \times\left(b_{m n}^{(p)^{2}}+c_{m n}^{(p)^{2}}\right)\right]\right\}}
$$


Table 1

Material properties of sandwich plate

\begin{tabular}{lcccc}
\hline Material & Young's modulus $(\mathrm{GPa})$ & Poisson's ratio & Density $\left(\mathrm{kg} \mathrm{m}^{-3}\right)$ & Coefficient of thermal expansion $\left({ }^{\circ} \mathrm{C}^{-1}\right)$ \\
\hline Facing & $70(1+0.001 \mathrm{i})$ & 0.3 & 2700 & $2.3 \mathrm{E}-5$ \\
Core & $7(1+0.001 \mathrm{i})$ & 0.3 & 1000 & $1.8 \mathrm{E}-5$ \\
\hline
\end{tabular}

Table 2

Comparisons of natural frequencies

\begin{tabular}{cccccccc}
\hline Modes & \multicolumn{2}{c}{ Without temperature load } & & \multicolumn{3}{c}{ With temperature load of $50^{\circ} \mathrm{C}$} \\
\cline { 2 - 3 } & $\begin{array}{c}\text { Numerical } \\
(\mathrm{Hz})\end{array}$ & $\begin{array}{c}\text { Analytical } \\
(\mathrm{Hz})\end{array}$ & $\begin{array}{c}\text { Error } \\
(\%)\end{array}$ & & $\begin{array}{c}\text { Numerical } \\
(\mathrm{Hz})\end{array}$ & $\begin{array}{c}\text { Analytical } \\
(\mathrm{Hz})\end{array}$ & $\begin{array}{c}\text { Error } \\
(\%)\end{array}$ \\
\hline$(1,1)$ & 364.50 & 371.26 & 1.85 & & 245.56 & 255.44 & 4.02 \\
$(2,1)$ & 752.84 & 765.49 & 1.68 & & 645.10 & 659.75 & 2.27 \\
$(1,2)$ & 1060.2 & 1067.5 & 0.69 & & 955.35 & 963.44 & 0.85 \\
$(3,1)$ & 1394.9 & 1407.8 & 0.93 & & 1290.6 & 1304.5 & 1.07 \\
$(2,2)$ & 1424.1 & 1450.0 & 1.82 & & 1318.9 & 1346.7 & 2.11 \\
$(3,2)$ & 2033.5 & 2074.0 & 1.99 & & 1928.3 & 1970.6 & 2.19 \\
$(1,3)$ & 2182.8 & 2183.2 & 0.02 & & 2079.4 & 2079.7 & 0.02 \\
$(4,1)$ & 2270.2 & 2278.3 & 0.36 & & 2166.4 & 2174.8 & 0.39 \\
\hline
\end{tabular}

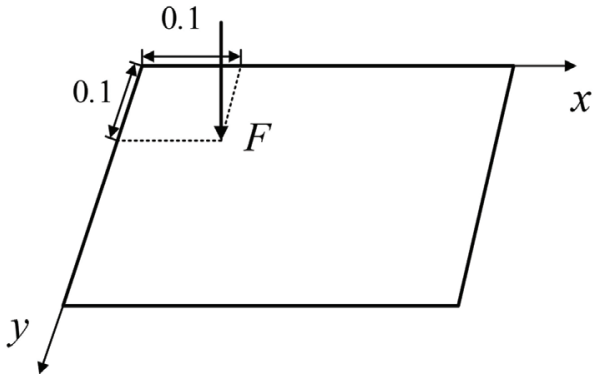

Fig. 2. Location of load point.

According to the equations above, the displacement responses can be acquired as Eq. (27):

$$
w(x, y, t)=\sum_{m} \sum_{n} \sum_{p} \sin \frac{m \pi x}{a} \sin \frac{n \pi y}{b} \frac{q_{0} \sin \frac{m \pi x_{0}}{a} \sin \frac{n \pi y_{0}}{b} \mathrm{e}^{\mathrm{i} \omega t}}{\left(\omega_{m n}^{(p) 2}-\omega^{2}\right)\left[\frac{a b}{4} \times\left(\rho h+\rho J \times\left(b_{m n}^{(p)^{2}}+c_{m n}^{(p)^{2}}\right)\right)\right]}
$$

The velocity can be found by taking derivative with respect to $t$ as Eq. (28).

$$
v(x, y, t)=\sum_{m} \sum_{n} \sum_{p} \sin \frac{m \pi x}{a} \sin \frac{n \pi y}{b} \frac{\mathrm{i} \omega q_{0} \sin \frac{m \pi x_{0}}{a} \sin \frac{n \pi y_{0}}{b} \mathrm{e}^{\mathrm{i} \omega t}}{\left(\omega_{m n}^{(p) 2}-\omega^{2}\right)\left[\frac{a b}{4} \times\left(\rho h+\rho J \times\left(b_{m n}^{(p)^{2}}+c_{m n}^{(p)^{2}}\right)\right)\right]}
$$

These equations indicate the amplitudes of displacement and velocity at one point are proportional, which can be clearly seen in the following Section 4. In this study, the vibro-acoustic coupling is neglected, i.e. the effect of sound radiation on the vibration of plates is ignored. By using the Rayleigh's integral [16], the sound pressure at the observation point $\left(x_{p}, y_{p}, z_{p}\right)$ above the plate can be acquired as Eq. (29):

$$
p\left(x_{p}, y_{p}, z_{p}, t\right)=\frac{\mathrm{i} \omega \rho_{0}}{2 \pi} \mathrm{e}^{\mathrm{i} \omega t} \int_{\Omega} \frac{v(x, y) \cdot \mathrm{e}^{-\mathrm{i} k R}}{R} \mathrm{~d} A
$$

where $k$ is the wave number which is evaluated by $\frac{\omega}{c}, c$ is the speed of sound, $\rho_{0}$ is the density of the fluid (air in the present paper), $R$ is the distance between points $(x, y, 0)$ on the plate and $\left(x_{p}, y_{p}, z_{p}\right)$ in the acoustic field and $\mathrm{d} A$ is the infinitesimal surface element of the area of the plate. Sound power $\bar{P}(\omega)$ radiated into the semi-infinite space above the plate can be expressed as Eq. (30):

$$
\bar{P}(\omega)=\frac{1}{2} \int_{\Omega} \operatorname{Re}\left\{v(x, y, \omega)^{*} p(x, y, 0, \omega)\right\} \mathrm{d} A
$$

The radiation efficiency [17] is defined as Eq. (31):

$$
e=\bar{P}(\omega) / \rho_{0} c a b\left\langle|v(\omega)|^{2}\right\rangle
$$

where $\left\langle|v(\omega)|^{2}\right\rangle$ is the spatially averaged mean-square normal velocity of the plate. 


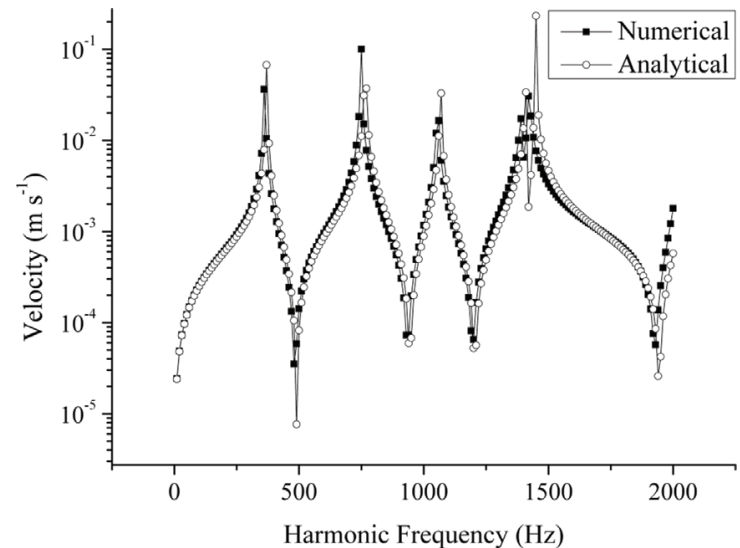

(a) Velocity

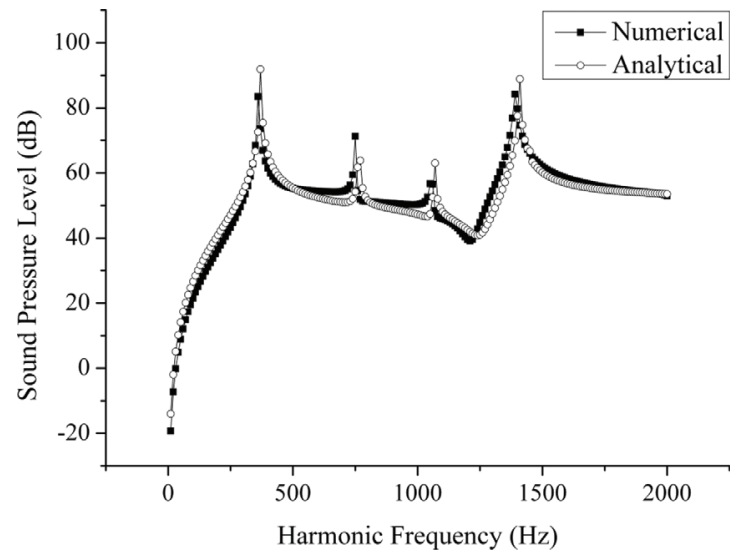

(b) Sound pressure level

Fig. 3. Comparisons of dynamic responses $\Delta T=0^{\circ} \mathrm{C}$.

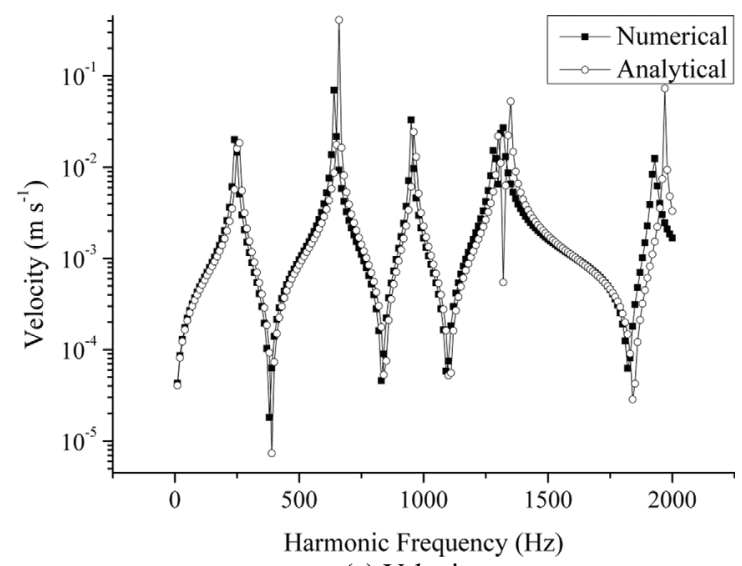

(a) Velocity

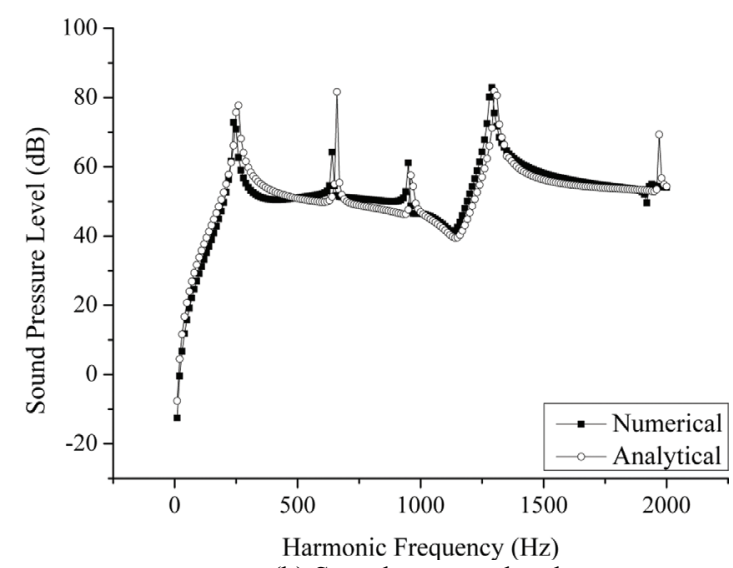

(b) Sound pressure level

Fig. 4. Comparisons of dynamic responses $\Delta T=50^{\circ} \mathrm{C}$.

\section{Validation}

To test the validity of the analytical solution, numerical analyses are carried out. A simply supported rectangular sandwich plate with dimensions of $400 \times 300 \times 10 \mathrm{~mm}$ is considered here. Two facings with a thickness of $0.5 \mathrm{~mm}$ and the core with a thickness of $9 \mathrm{~mm}$ are made of two kinds of isotropic materials respectively. The properties for these materials are listed in Table 1 . In the present study, the structural damping ratio 0.001 is taken into consideration.

\subsection{Validation for natural frequencies and modes}

Commercial software Nastran is used for testing the natural frequencies and modes with FEM. In order to confirm the accuracy of the consideration of thermal load, natural frequencies are obtained with and without temperature load respectively. The comparisons of the analytical results with those from Nastran are shown in Table 2, in which the first eight frequencies and modes are presented.

It can be clearly seen that the analytical solutions of both natural frequencies and modes match well with the results given by FEM, no matter whether the thermal environment exists. 


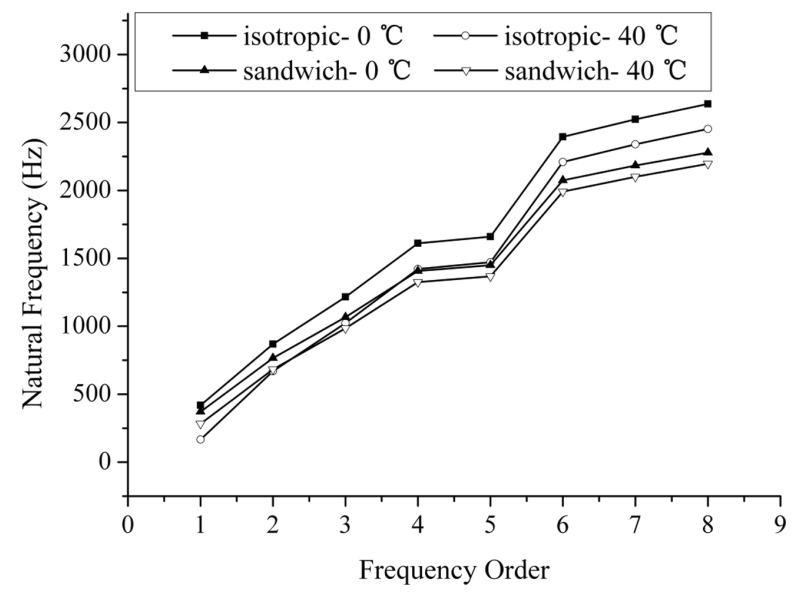

Fig. 5. Comparisons of natural frequency.

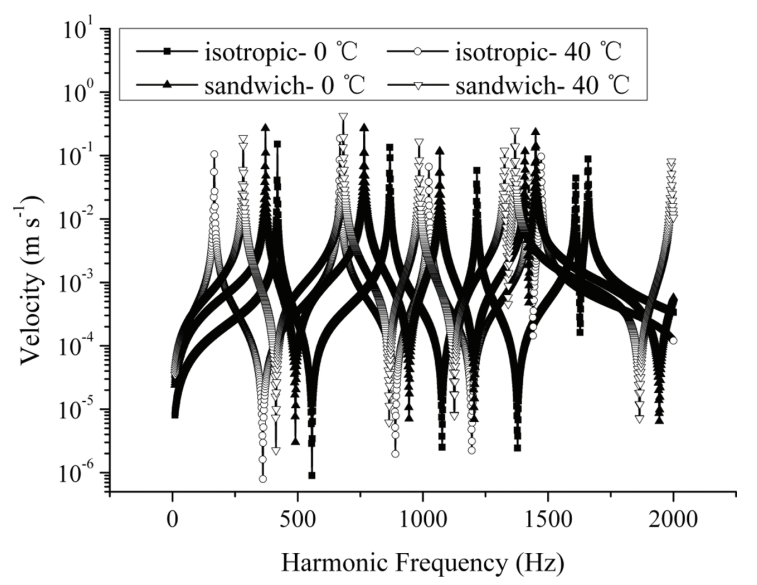

(a) Velocity

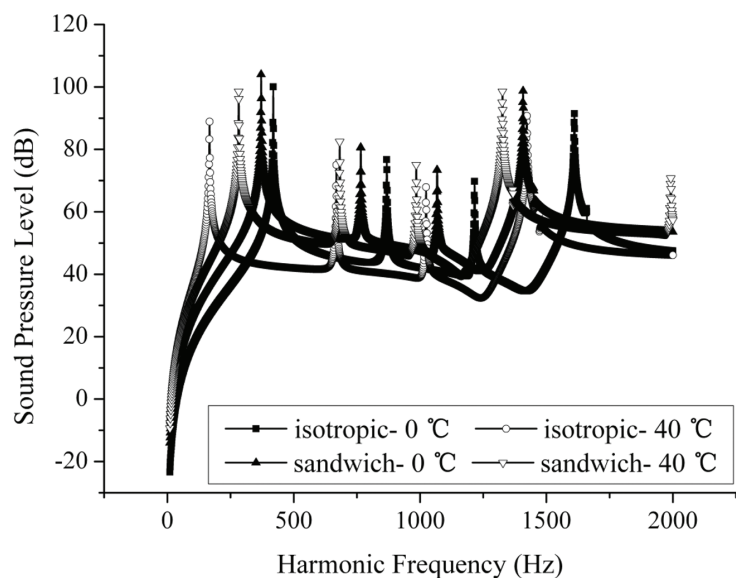

(b) Sound pressure level

Fig. 6. Dynamic responses.

\subsection{Validation for vibration and acoustic response}

A concentrated harmonic load with the amplitude of $1 \mathrm{~N}$ is applied to the plate. The location of the load point is shown in Fig. 2. The plate is assumed to be vibrating in the air whose density is $\rho_{0}=1.2 \mathrm{~kg} / \mathrm{m}^{3}$ with a speed of sound $c=340 \mathrm{~m} / \mathrm{s}$. A frequency range of $10-2000 \mathrm{~Hz}$ for the harmonic load is chosen for the dynamic analysis.

Numerical velocity responses caught at $(0.1 \mathrm{~m}, 0.1 \mathrm{~m}, 0 \mathrm{~m})$ and sound pressure levels at the observation point $(0.1 \mathrm{~m}, 0.1 \mathrm{~m}, 3 \mathrm{~m})$ are obtained by commercial software VA one with FEM/BEM.

From Figs 3 and 4, one can find that whether the plate is under thermal environment or not, the analytical vibration response agrees with that of VA one very well, while there are slight differences between sound pressure levels. So far, it could be believable that the present analytical solution is correct based on the above mutual validation.

\section{Analytical results and discussion}

A rectangular simply supported sandwich plate is now considered for the detailed investigation. The material properties and dimensions of the plate have been shown above. For the purpose of making the response figures present enough information clearly, the forced frequency domain should be considered in the follow. From Table 2 , 
Table 3

Natural frequencies under thermal loads

\begin{tabular}{crcc}
\hline Modes & \multicolumn{3}{c}{ Natural frequencies (Hz) } \\
\cline { 2 - 4 } & $\Delta T=0^{\circ} \mathrm{C}$ & $\Delta T=50^{\circ} \mathrm{C}$ & $\Delta T=90^{\circ} \mathrm{C}$ \\
\hline$(1,1)$ & 371.26 & 255.44 & 84.738 \\
$(2,1)$ & 765.49 & 659.75 & 560.98 \\
$(1,2)$ & 1067.49 & 963.44 & 871.31 \\
$(3,1)$ & 1407.8 & 1304.5 & 1215.5 \\
$(2,2)$ & 1450.0 & 1346.7 & 1257.9 \\
$(3,2)$ & 2074.0 & 1970.6 & 1883.8 \\
$(1,3)$ & 2183.2 & 2079.7 & 1993.1 \\
$(4,1)$ & 2278.3 & 2174.8 & 2088.3 \\
\hline
\end{tabular}

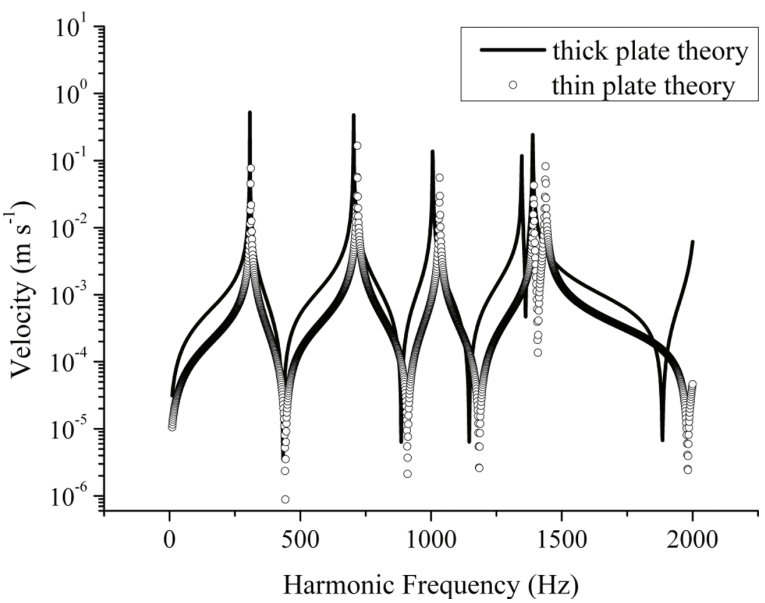

(a) Velocity

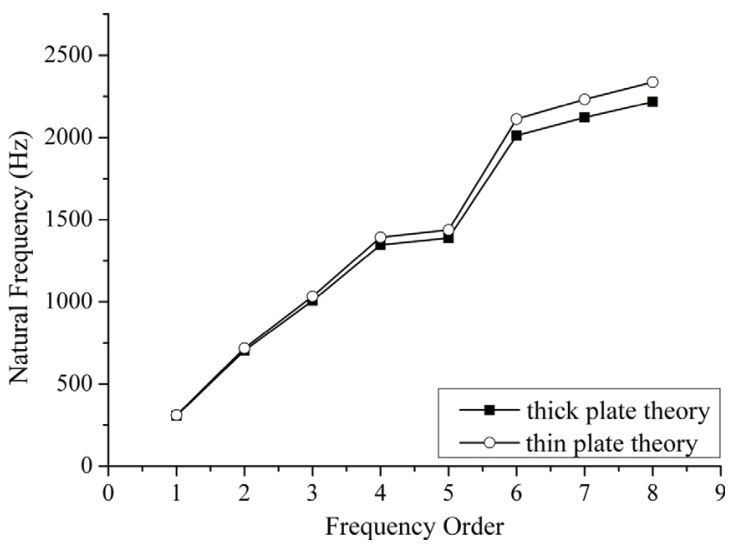

Fig. 7. Comparisons of natural frequency.

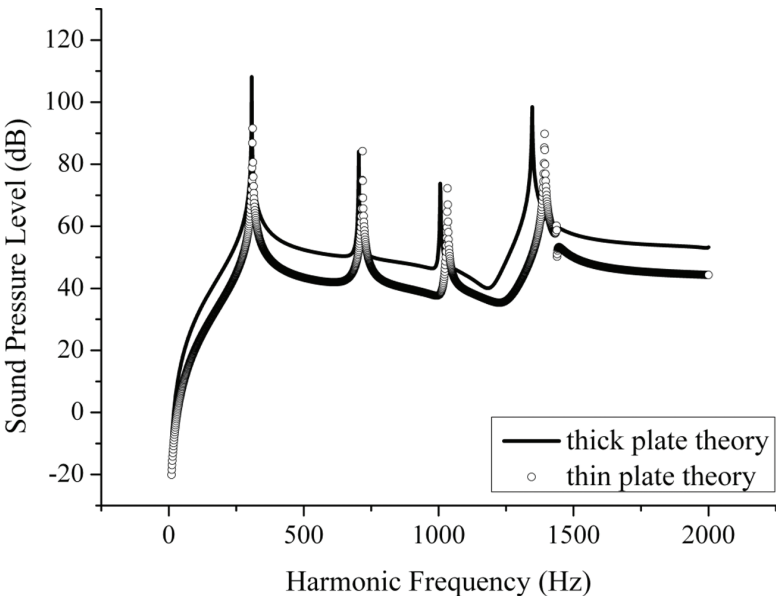

(b) Sound pressure level

Fig. 8. Dynamic responses.

one can see that, in the range of $10-2000 \mathrm{~Hz}$, there are about five natural frequencies, which means there will be about five peaks on each curve in the response figures. So a frequency range of $10-2000 \mathrm{~Hz}$ is chosen in dynamic analysis. In order to meet the requirement of mode superposition method, the natural frequency is calculated up to $4000 \mathrm{~Hz}$

The force, structural damping ratio and the observation points for vibration and acoustic response are all the same as those in Section 3.

\subsection{Buckling temperature}

In this paper, the vibration and acoustic responses of sandwich plate have been analyzed by assuming that the plate is subjected to a uniform temperature distribution. By the approach mentioned in Section 2, the critical buckling temperature for the plate is verified to be $94^{\circ} \mathrm{C}$. Once the temperature load exceeds the critical buckling temperature, the responses can't be considered with linear methods. The uniform temperature applied on the plate is varied from $0^{\circ} \mathrm{C}$ to $90^{\circ} \mathrm{C}$ for the natural and dynamic structural analyses.

\subsection{Difference between isotropic plate and sandwich plate}

The specific characteristics of the sandwich plate are discussed in this section. The comparisons of the sandwich plate and the isotropic plate are carried out to show the difference caused by sandwich structure. Here we discuss 


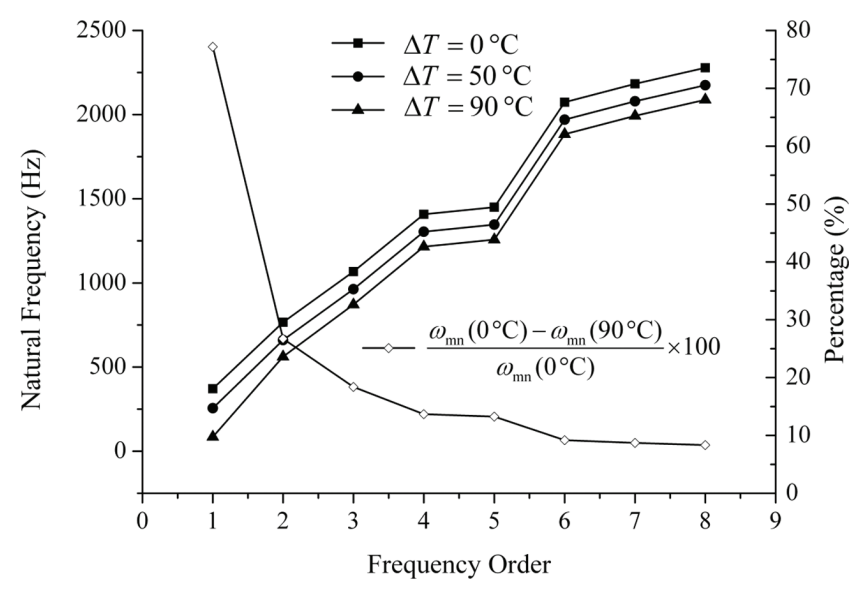

Fig. 9. Natural frequencies and their relative difference.

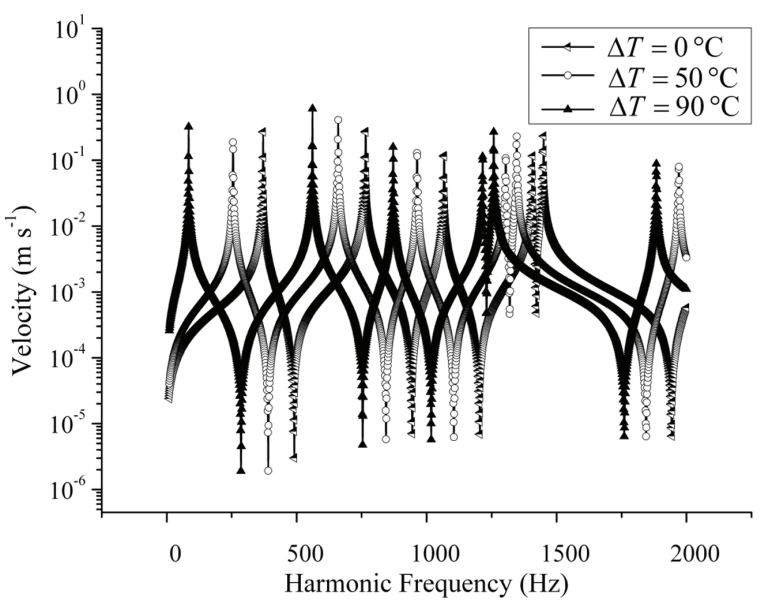

Fig. 11. Velocity.

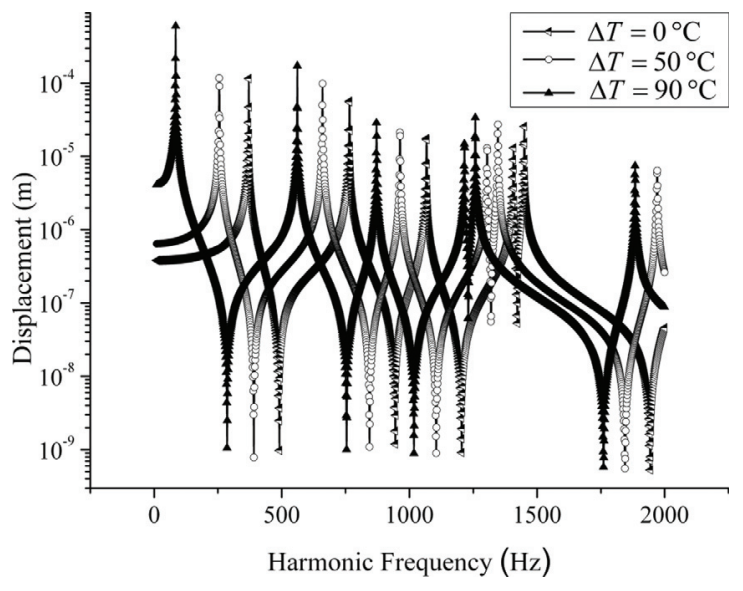

Fig. 10. Displacement.

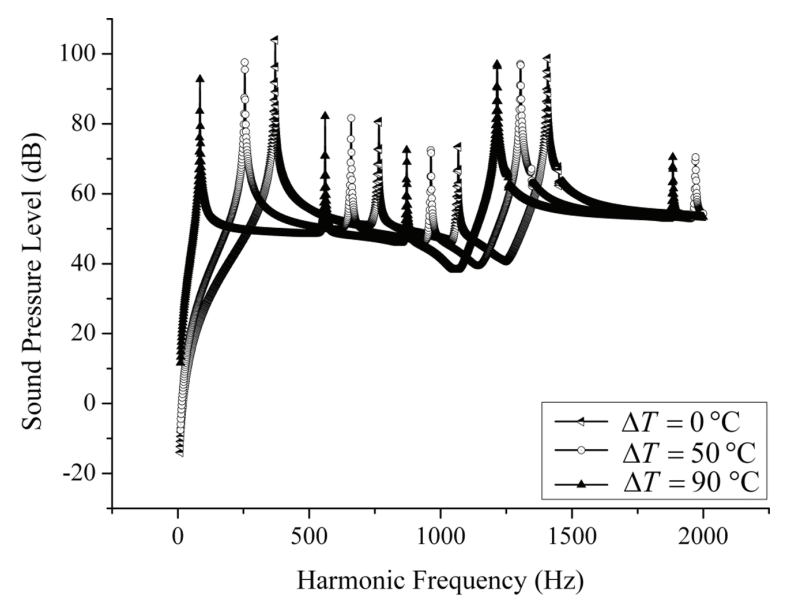

Fig. 12. Sound pressure level.

the difference between the sandwich plate of this paper and the isotropic plate with the same material as the facing of the sandwich plate.

From Fig. 5, it is found that natural frequencies of the sandwich plate are smaller than those of the isotropic plate without thermal environment. The difference between them is bigger in high-frequency domain. The thermal load reduces the natural frequencies of both of them, but doesn't change the slope of each curve. The influence caused by thermal load is more obvious on isotropic plate than sandwich plate.

When the plates are subjected to a concentrated force, the sandwich plate has bigger peak responses than isotropic plate, which are shown in Fig. 6. Due to the difference of the natural frequencies, the peak frequencies of sandwich plate are much closer.

\subsection{Thick plate theory and thin plate theory}

As mentioned in the introduction, the derivations in the present work are based on thick plate theory. In order to show the necessity of considering the effects of the shear deformation and rotational inertia, the natural frequencies and vibration responses obtained through thick plate theory and thin plate theory are shown in Figs 7 and 8 .

The trend can be clearly seen that the natural frequencies calculated from thick plate theory are smaller than the ones from thin plate theory. The difference between thick and thin plate theories gets bigger in high-frequency domain as shown in Fig. 7. 


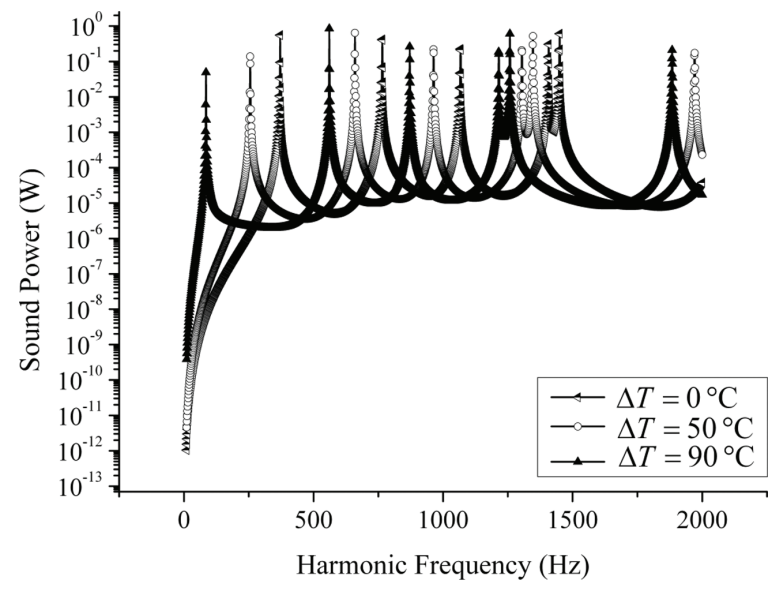

Fig. 13. Sound power.

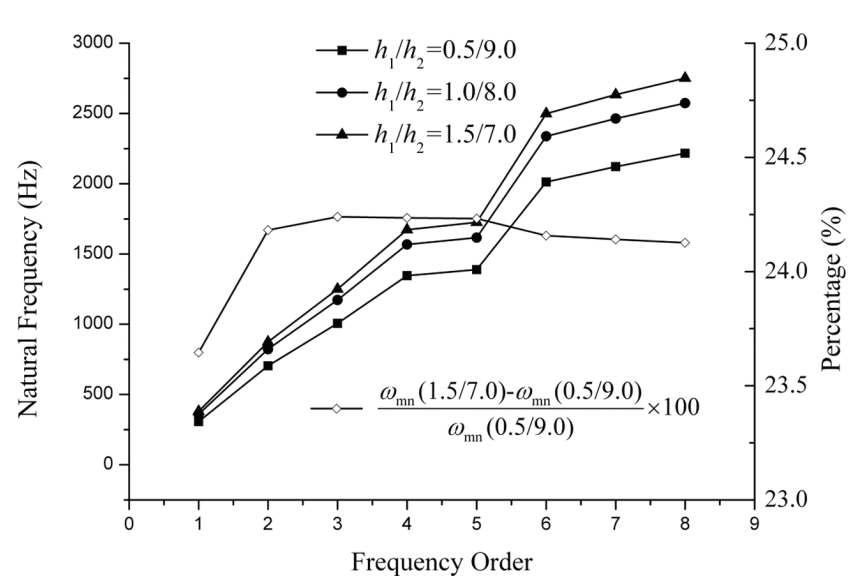

Fig. 15. Natural frequencies and their relative difference.

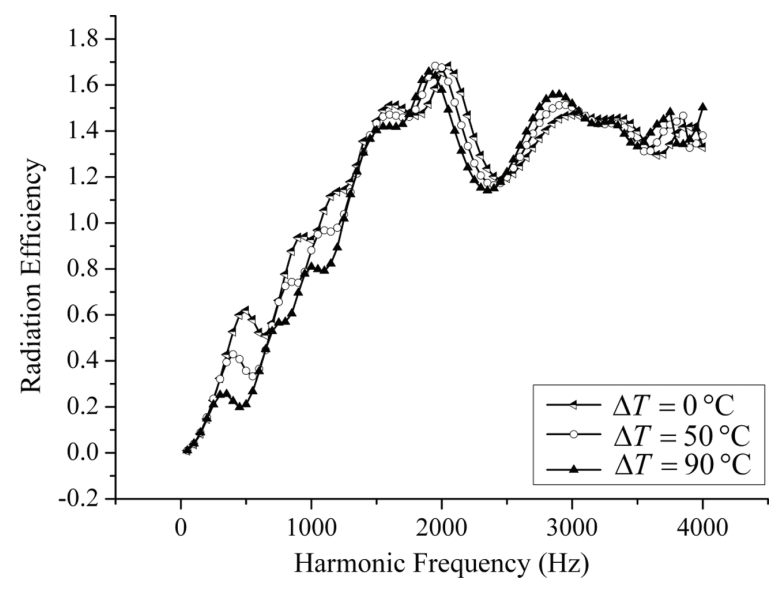

Fig. 14. Radiation efficiency.

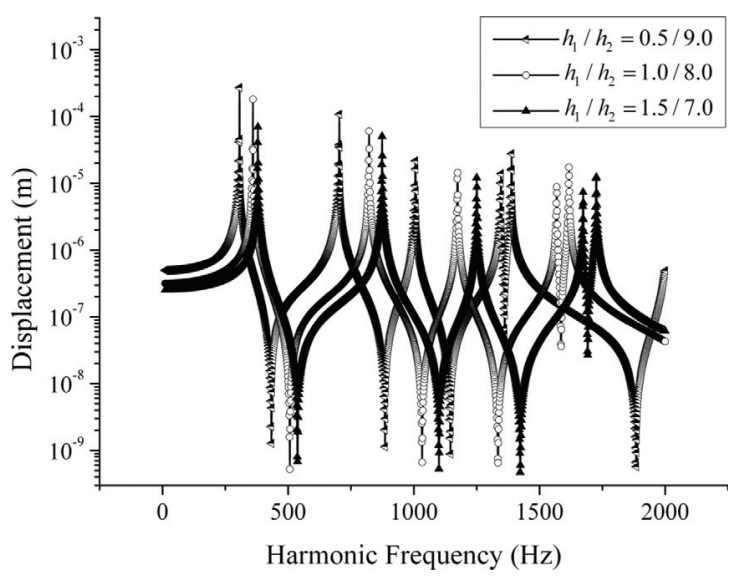

Fig. 16. Displacement.

Synthesizing the results shown in Figs 7 and 8, we can see that thin plate theory overrates the natural frequency and underrates the dynamic response and the peaks in high frequency range shift to low frequency using thick plate theory.

\subsection{Different temperatures}

In this section, $\Delta T=0^{\circ} \mathrm{C}, 50^{\circ} \mathrm{C}, 90^{\circ} \mathrm{C}$ are imposed on the sandwich plate. The first eight frequencies are shown in Table 3, from which one can observe the trend that the natural frequencies reduce with the temperature rising. The modes for the plate are almost the same under different temperature. As indicated in Eqs (1) and (9), the effect of temperature is treated as an in-plane traction, which is equivalent to a decrease in stiffness of the sandwich plates. This softening effect lowers the natural frequencies, while preserves the mode shapes if the boundary condition and the structure keep invariant.

From Fig. 9, we can see that the relative frequency difference of the first natural frequency is much greater than others, which means the effect induced by thermal load is more obvious in fundamental frequency.

The vibration responses at load point and the sound pressure level responses at observation point are shown in Figs 10-12. The sound power and radiation efficiency of the plate calculated by sound pressure and velocity are presented in Figs 13 and 14. 


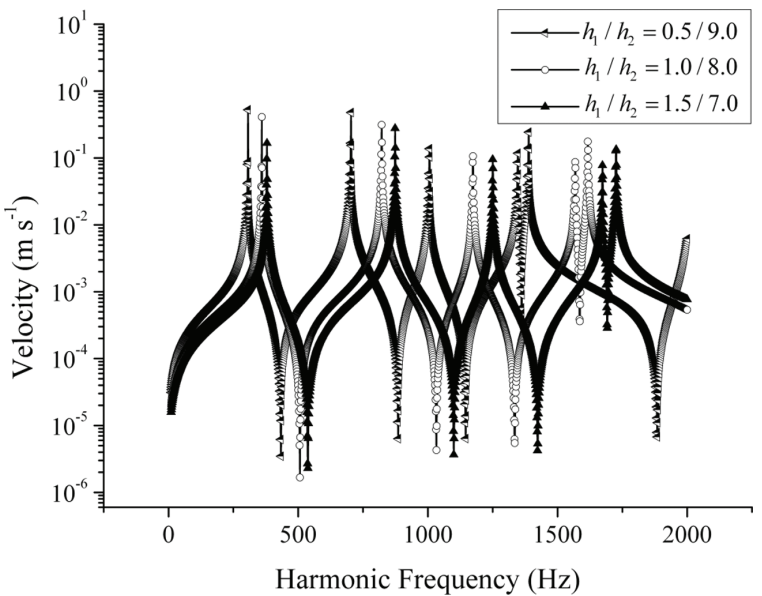

Fig. 17. Velocity.

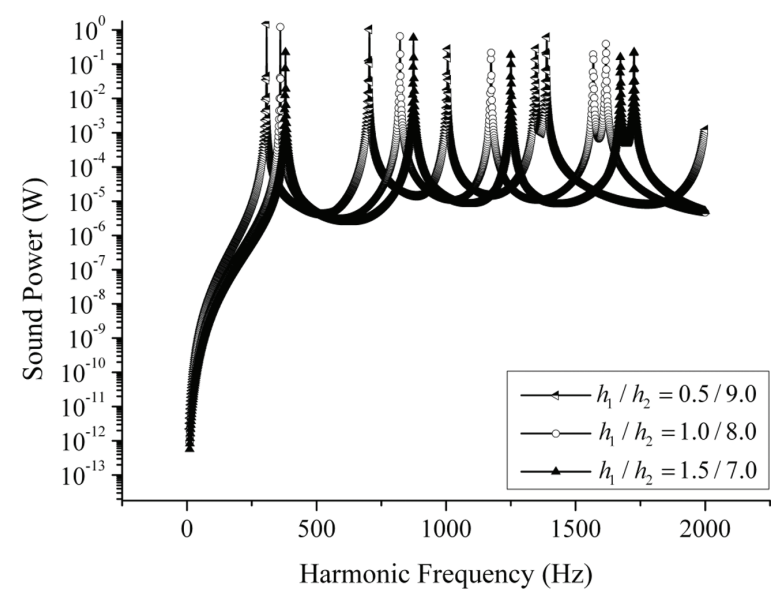

Fig. 19. Sound power.

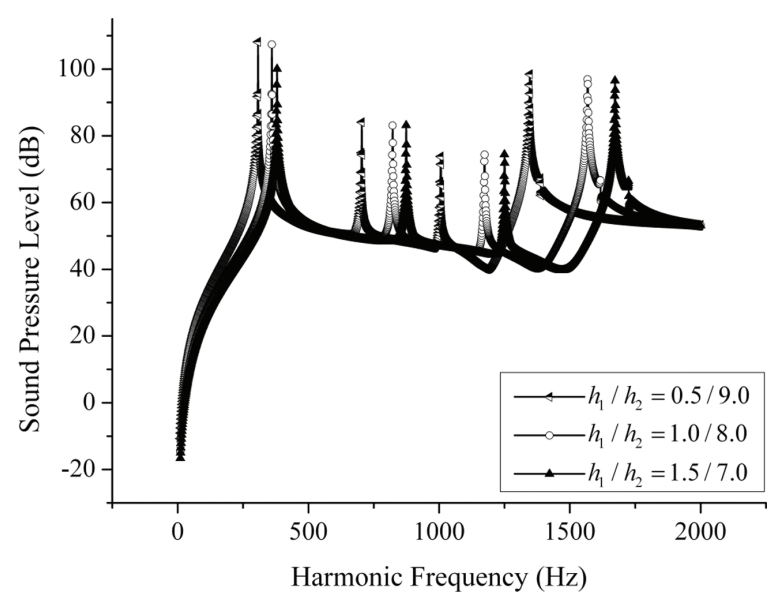

Fig. 18. Sound pressure level.

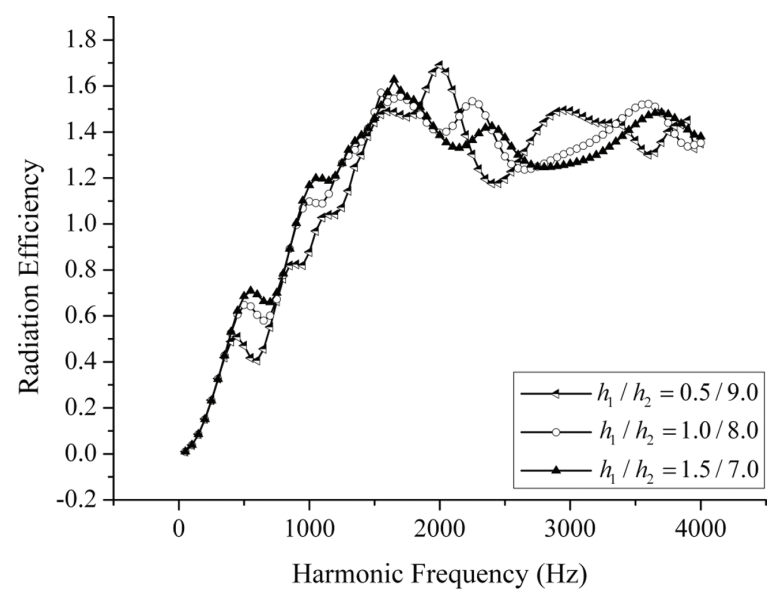

Fig. 20. Radiation efficiency.

One can observe that there are about five peaks on each curve, which are corresponding to natural frequencies in the range of 10-2000 Hz. With the thermal load increasing, the peaks of vibration and acoustic responses move leftward in these figures, which is induced by the decrease of natural frequencies.

As the thermal load increases, the peak of displacement tends to increase. However, the peak of velocity partially decreases in low-frequency domain. From Eq. (28), one can see that the value of velocity is calculated through multiplying displacement by corresponding forced frequency. When the value and the frequency of peak for displacement response have the same trend of increasing or decreasing, the relative difference in velocity will get bigger, and conversely it will get smaller or even reverse. In Fig. 10, the frequency of peak reduces and the value of peak increases as temperature rises, which means that the tendency of rising in displacement is weakened or reversed in velocity. From Fig. 9, one can observe that the effect induced by the thermal load is more significant in fundamental frequency, so the trend doesn't reverse in the high-frequency range.

SPL (Sound pressure level) is calculated by Eq. (29), from which we can infer the trend of SPL is similar with that of velocity. While the temperature load increases, the peaks float to low-frequency range.

The sound power has the same trend as the SPL. Radiation efficiency, which shows the radiating effectiveness of the plate, is acquired by Eq. (31). In about $10-300 \mathrm{~Hz}$, there is hardly difference among three curves. The first crest with smaller value appears at lower frequency as temperature rises. The efficiency rises rapidly in low-frequency and becomes flat when the harmonic frequency reaches about $2000 \mathrm{~Hz}$. 
Table 4

Natural frequencies with different thickness ratios

\begin{tabular}{cccc}
\hline Modes & \multicolumn{3}{c}{ Natural frequencies $(\mathrm{Hz})$} \\
\cline { 2 - 4 } & $h_{1}=0.5 \mathrm{~mm}$ & $h_{1}=1 \mathrm{~mm}$ & $h_{1}=1.5 \mathrm{~mm}$ \\
& $h_{2}=9 \mathrm{~mm}$ & $h_{2}=8 \mathrm{~mm}$ & $h_{2}=7 \mathrm{~mm}$ \\
\hline$(1,1)$ & 307.05 & 359.98 & 379.65 \\
$(2,1)$ & 703.96 & 822.24 & 874.18 \\
$(1,2)$ & 1006.4 & 1173.6 & 1250.3 \\
$(3,1)$ & 1346.8 & 1568.3 & 1673.2 \\
$(2,2)$ & 1389.0 & 1617.2 & 1725.5 \\
$(3,2)$ & 2012.6 & 2337.9 & 2498.8 \\
$(1,3)$ & 2121.7 & 2463.7 & 2633.9 \\
$(4,1)$ & 2216.8 & 2573.2 & 2751.6 \\
\hline
\end{tabular}

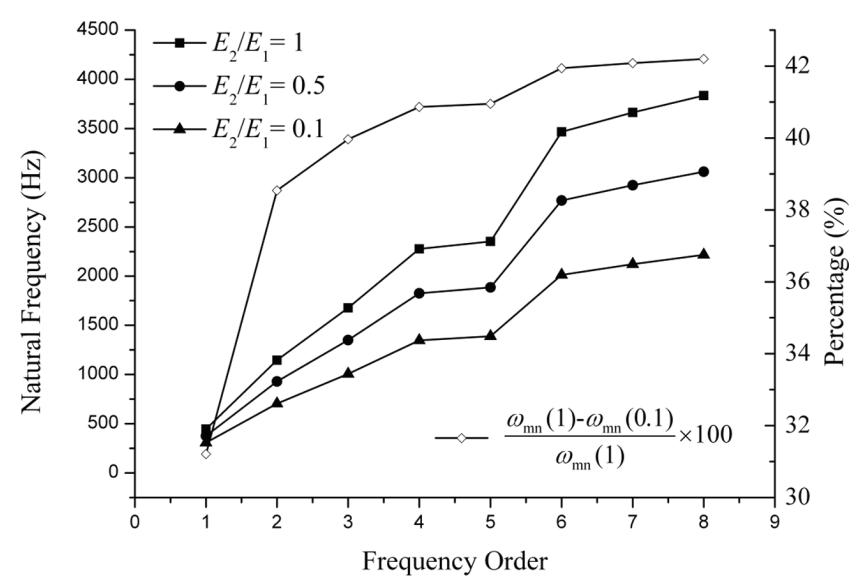

Fig. 21. Natural frequencies and their relative difference.
Table 5

Natural frequencies with different Young's moduli of core

\begin{tabular}{cccc}
\hline Modes & \multicolumn{3}{c}{ Natural frequencies (Hz) } \\
\cline { 2 - 4 } & $E_{1}=70 \mathrm{GPa}$ & $E_{1}=70 \mathrm{GPa}$ & $E_{1}=70 \mathrm{GPa}$ \\
& $E_{2}=70 \mathrm{GPa}$ & $E_{2}=35 \mathrm{GPa}$ & $E_{2}=7 \mathrm{GPa}$ \\
\hline$(1,1)$ & 446.36 & 376.00 & 307.05 \\
$(2,1)$ & 1145.4 & 928.61 & 703.96 \\
$(1,2)$ & 1676.2 & 1349.8 & 1006.4 \\
$(3,1)$ & 2277.4 & 1826.6 & 1346.8 \\
$(2,2)$ & 2352.2 & 1885.9 & 1389.0 \\
$(3.2)$ & 3466.5 & 2768.8 & 2012.6 \\
$(1,3)$ & 3663.2 & 2924.5 & 2121.7 \\
$(4,1)$ & 3835.0 & 3060.4 & 2216.8 \\
\hline
\end{tabular}

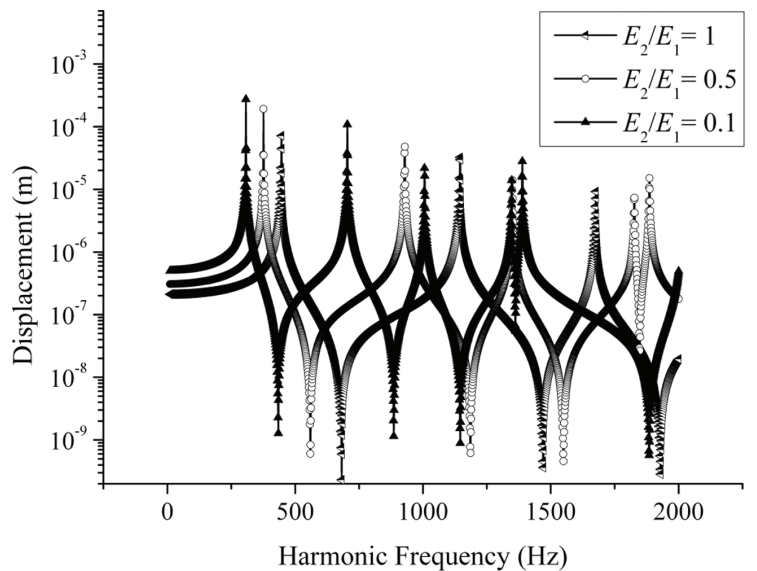

Fig. 22. Displacement.

\subsection{Different thickness ratios of facing to core}

Plates with different thickness ratios of facing to core present different characteristics. In this section, we will discuss them with three conditions of different thickness ratios under temperature load of $30^{\circ} \mathrm{C}$. The aggregate thickness remains $10 \mathrm{~mm}$. The natural frequencies and modes are shown in Table 4 . The trend that the frequencies raise with the thickness ratio increasing can be seen evidently.

Dynamic analysis is also conducted. The vibration and acoustic responses are shown in Figs 16-20. As the thickness ratio of facing to core gets bigger, peaks of the displacement become smaller and float to the high-frequency domain. The relative peak differences in velocity are weakened slightly. However, they are of the same trend. As shown in Fig. 15, the relative differences of frequency are about $24 \%$ for the first five frequencies. So the resonant frequency, which is the frequency of peak in the figures, goes further from each other when forced frequency increases because of superposition difference.

As thickness ratio increases, the peak of SPL reduces and moves rightward in the response figure. The sound power has the same trend as the SPL. In low-frequency range, three radiation efficiency curves are overlapping. The first crest with bigger value appears at higher frequency as ratio increases. The curve becomes smoother while the ratio gets bigger.

\subsection{Different Young's moduli of core}

In this section, we will show the specific mechanical characteristics of sandwich plates with hard or soft core. Three Young's moduli of core are chosen under temperature load of $30^{\circ} \mathrm{C}$. The Young's modulus of facings $E_{1}$ keeps unchanged. The first eight frequencies of plate with different Young's moduli of core are shown in Table 5. As $E_{2}$ gets smaller, the natural frequencies become smaller and the mode shapes remain the same. The differences 


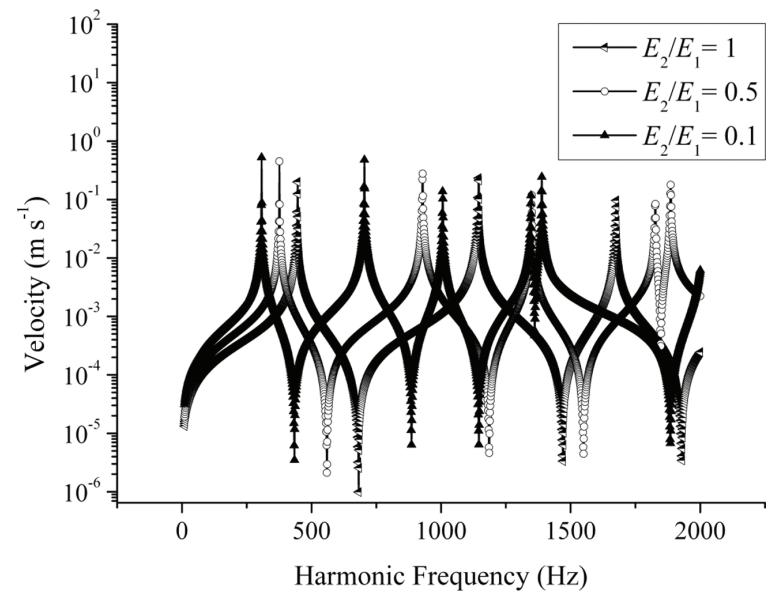

Fig. 23. Velocity.

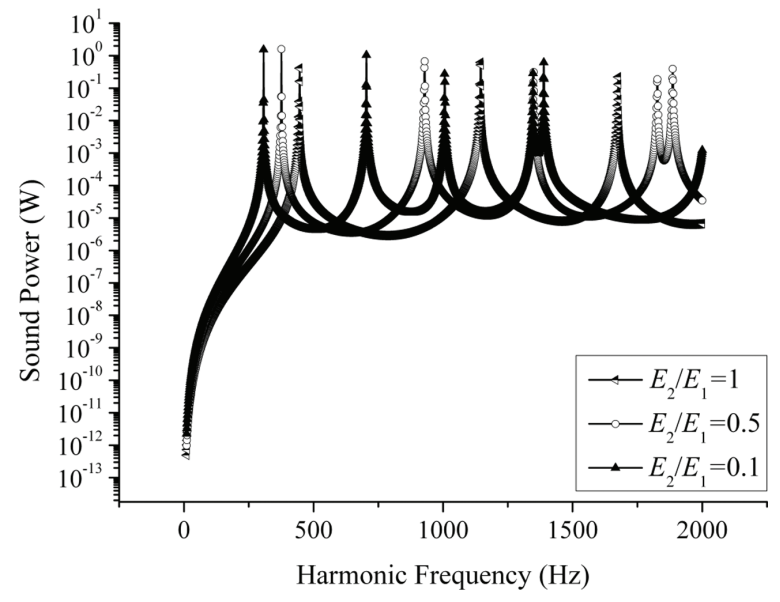

Fig. 25. Sound power.

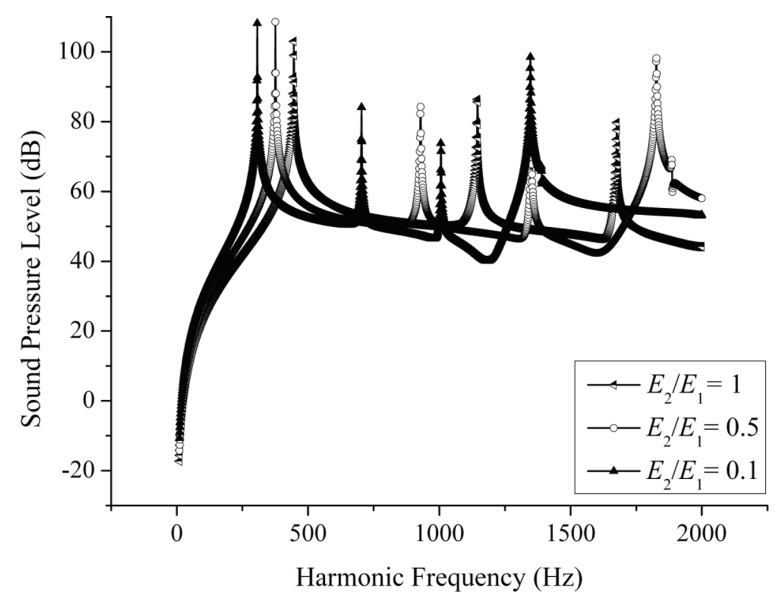

Fig. 24. Sound pressure level.

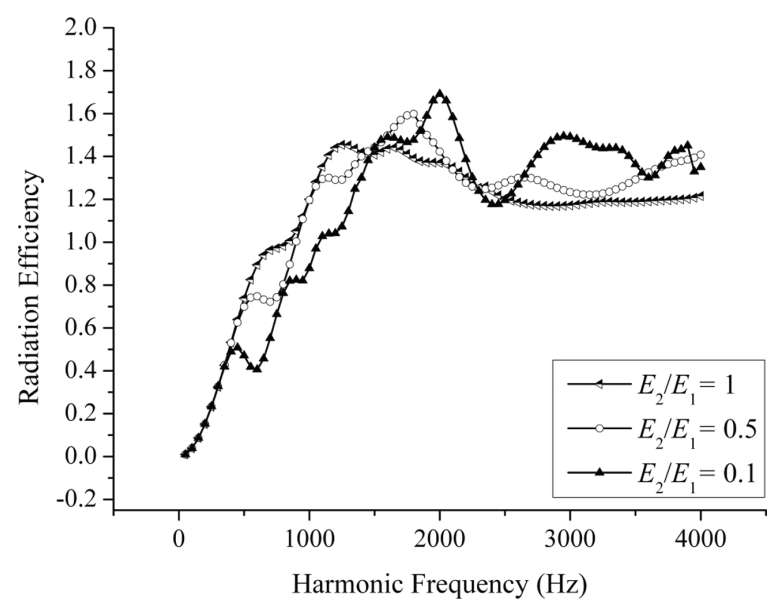

Fig. 26. Radiation efficiency.

among three curves are more remarkable in high-frequency domain, which means the Young's modulus has more influence in high-frequency region as Fig. 21 shows.

Dynamic responses of the plate with different Young's moduli of core are shown in Figs 22-26. From Fig. 22, one can find that when the Young's modulus of core decreases, the peaks of displacement become higher and float to low-frequency range. However, this tendency is weakened slightly in the velocity response.

SPL, as well as sound power, has a similar tendency that the peaks float to low-frequency range when Young's modulus reduces. Radiation efficiency response curve becomes more volatile when the Young's modulus of core decreases. It can be clearly seen that there is almost no influence impacted on the radiation efficiency of the plate in low-frequency range. When the Young's modulus of core reduces, the first efficiency crest becomes smaller with a lower forced frequency.

\section{Conclusion}

Sandwich plates usually have smaller natural frequencies and are less influenced by thermal environment than isotropic plates. Neglecting the effects of the shear deformation and rotational inertia, the thin plate theory overrates the natural frequency and underrates the dynamic response generally. 
The effects induced by thermal environment on the vibration response and consequent sound radiation are obtained for a sandwich plate under different conditions. Sandwich plates subjected to different thermal loads present different characteristics. The rise of temperature load decreases the natural frequencies and moves the peaks of response to low-frequency range. Thermal environment influences the low natural frequency remarkably, while the effect is less obvious in high-frequency domain. The displacement peaks increase as the temperature load rises, while this tendency is partially reversed in the velocity response. SPL has a similar trend with the velocity. The radiation efficiency has no change in the low-frequency region, and the first crest with a smaller value comes out in a lower forced frequency when the thermal load increases.

Keeping the total thickness unchanged, the natural frequency is enlarged while the thickness ratio of facing to core is increasing. It also makes the peaks of displacement reduce and float to the high-frequency region. The trend is weakened in the velocity response. The variation trends of SPL and sound power response are quite similar with that of velocity. Three radiation efficiency curves are overlapping in the low-frequency region, and the curve becomes smoother when the ratio gets bigger.

Making Young's modulus of facing remains the same and decreasing Young's modulus of the core can cause the decrement of the natural frequencies, which makes response curves move to the low-frequency range. The influence imposed by the change of Young's modulus of core is more remarkable in high-frequency domain. The displacement peaks increase when the modulus gets smaller, and this tendency is weakened slightly in the velocity response. SPL and sound power response have quite similar variation trend with that of the velocity. The radiation efficiency curve becomes more volatile as the modulus decreases.

\section{Acknowledgment}

The authors are grateful to the National Natural Science Foundation of China (Grant No. 91016008, 11021202).

\section{Appendix A}

The basic assumptions of non-classical theory for medium and thick plate, which considers the effects of shear deformation, rotational inertia, and compressional deformation, are adopted to derive the following governing equations and equivalent transformation relations. The composite plate, which is heterogeneous along the thickness, is transformed to an equivalent homogeneous anisotropic plate.

Considering a composite plate with uniform thickness, the elastic constants $e_{i j}(z)$ and mass density $\rho(z)$ are functions of the coordinate $z$ along the thickness, while there is an elastic symmetric plane, which is parallel to the middle surface, for each component layer. According to the assumptions of non-classical theory, the in-plane displacements are expressed as follows:

$$
\begin{aligned}
& u(x, y, z, t)=-\frac{\partial w}{\partial x} z+\psi_{x}(x, y, t) f(z) \\
& v(x, y, z, t)=-\frac{\partial w}{\partial y} z+\psi_{y}(x, y, t) f(z)
\end{aligned}
$$

where the second terms on the right-hand sides are introduced to take the shear deformation into account. $\psi_{x}$ and $\psi_{y}$ are generalized shear rotations, while $f(z)$ is the displacement distribution function of shear deformation. The strain components are given according to the general strain-displacement relations:

$$
\begin{array}{cl}
\varepsilon_{x}=-\frac{\partial^{2} w}{\partial x^{2}} z+\frac{\partial \psi_{x}}{\partial x} f(z) & \varepsilon_{y}=-\frac{\partial^{2} w}{\partial y^{2}} z+\frac{\partial \psi_{y}}{\partial y} f(z) \\
\gamma_{x y}=-2 \frac{\partial^{2} w}{\partial x \partial y} z+\left(\frac{\partial \psi_{x}}{\partial y}+\frac{\partial \psi_{y}}{\partial x}\right) f(z) & \gamma_{y z}=\psi_{y} f^{\prime}(z) \quad \gamma_{z x}=\psi_{x} f^{\prime}(z)
\end{array}
$$


The generalized Hooke's law determines the following stress-strain relations:

$$
\begin{aligned}
\sigma_{i} & =e_{i j}(z) \varepsilon_{j} & & (i, j=1,2,3,6) \\
\sigma_{k} & =e_{k l}(z) \varepsilon_{l} & & (k, l=4,5)
\end{aligned}
$$

where,

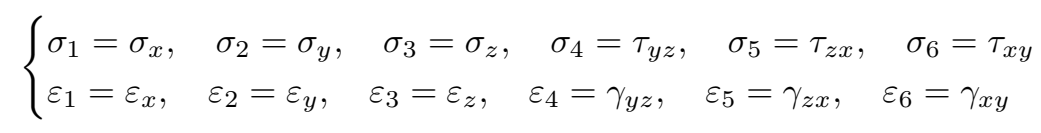

By taking the value $i=3$ in Eq. (A-3), the $\varepsilon_{3}$ can be solved. Substituting $\varepsilon_{3}$ into Eq. (A-3) and considering the assumptions of non-classical theory, the following formula can be obtained:

$$
\sigma_{i}=\left[e_{i \alpha}(z)-\frac{e_{i 3}(z)}{e_{33}(z)} e_{3 \alpha}(z)\right] \varepsilon_{\alpha}+\frac{e_{i 3}(z)}{e_{33}(z)} q(x, y, t) B(z) \quad(i, \alpha=1,2,6)
$$

where the second term on the right-hand side is generated by compressional deformation. $q$ is the external load, while $B(z)$ stands for the distribution function of compressional deformation.

Substituting the Eqs (A-4) and (A-6) into the expressions of internal forces on cross section, we can get Eqs (A-7) and (A-8):

$$
\begin{aligned}
Q_{k} & =\int_{-h / 2}^{h / 2} \sigma_{k} \mathrm{~d} z=\int_{-h / 2}^{h / 2}\left[e_{k 4}(z) \psi_{y} f^{\prime}(z)+e_{k 5}(z) \psi_{x} f^{\prime}(z)\right] \mathrm{d} z \\
M_{i} & =\int_{-\frac{h}{2}}^{\frac{h}{2}} \sigma_{i} z \mathrm{~d} z=D_{i \alpha} \frac{\partial \beta_{\alpha}}{\partial x_{\alpha}}+\frac{D_{i i} k_{\sigma}}{C_{i} h} q \quad(i, \alpha=1,2,6)
\end{aligned}
$$

where $D_{i \alpha}$ is the compositional stiffness coefficient:

$$
D_{i \alpha}=\int_{-h / 2}^{h / 2}\left[e_{i \alpha}(z)-\frac{e_{i 3}(z)}{e_{33}(z)} e_{3 \alpha}(z)\right] z^{2} \mathrm{~d} z \quad(i, \alpha=1,2,6)
$$

$\beta_{\xi}$ is the generalized rotation:

$$
\beta_{\xi}=-\frac{\partial w}{\partial x_{\xi}}+\frac{\psi_{\xi}}{h} k_{\tau} \quad(\xi=1,2)
$$

the derivative of $\beta_{6}$ is defined as:

$$
\frac{\partial \beta_{6}}{\partial x_{6}}=\frac{\partial \beta_{1}}{\partial x_{2}}+\frac{\partial \beta_{2}}{\partial x_{1}}
$$

$C_{i}$ is the compositional compression modulus:

$$
C_{i}=\frac{D_{i i} k_{\sigma}}{h}\left[\int_{-\frac{h}{2}}^{\frac{h}{2}} \frac{e_{i 3}(z)}{e_{33}(z)} B(z) z \mathrm{~d} z\right]^{-1} \quad(i=1,2,6)
$$

$k_{\tau}$ is the coefficient of shear deformation:

$$
k_{\tau}=\frac{h \int_{-\frac{h}{2}}^{\frac{h}{2}} K(z) f(z) z \mathrm{~d} z}{\int_{-\frac{h}{2}}^{\frac{h}{2}} K(z) z^{2} \mathrm{~d} z}
$$


$k_{\sigma}$ is the coefficient of compressional deformation:

$$
k_{\sigma}=\frac{12}{h^{2}} \int_{-\frac{h}{2}}^{\frac{h}{2}} B(z) z \mathrm{~d} z
$$

$K(z)$ is introduced in the formula $e_{i j}(z)=e_{i j}^{0} \cdot K(z)$ to represent the distribution function of material property. For different $K(z), k_{\tau}$ is figured out to be in the interval $k_{\tau} \approx 1.0 \sim 1.25$, thus it is commonly simplified as follows:

$$
k_{\tau}=\frac{h \int_{-\frac{h}{2}}^{\frac{h}{2}} \bar{K} f(z) z \mathrm{~d} z}{\int_{-\frac{h}{2}}^{\frac{h}{2}} \bar{K} z^{2} \mathrm{~d} z}=\frac{12}{h^{2}} \int_{-\frac{h}{2}}^{\frac{h}{2}} f(z) z \mathrm{~d} z
$$

where $\bar{K}$ is the arithmetic mean of $K(z)$.

The equilibrium equations considering membrane forces can be expressed as Eq. (A-16):

$$
\begin{aligned}
& \frac{\partial Q_{x}}{\partial x}+\frac{\partial Q_{y}}{\partial y}+N_{x} \frac{\partial^{2} w}{\partial x^{2}}+N_{y} \frac{\partial^{2} w}{\partial y^{2}}+2 N_{x y} \frac{\partial^{2} w}{\partial x \partial y}+q=\rho h \frac{\partial^{2} w}{\partial t^{2}} \\
& \frac{\partial M_{x}}{\partial x}+\frac{\partial M_{x y}}{\partial y}-Q_{x}=\rho J \frac{\partial^{2} \beta_{x}}{\partial t^{2}} \\
& \frac{\partial M_{x y}}{\partial x}+\frac{\partial M_{y}}{\partial y}-Q_{y}=\rho J \frac{\partial^{2} \beta_{y}}{\partial t^{2}}
\end{aligned}
$$

where the effect of rotational inertia results in the terms contain $\rho J$, while $\rho h$ and $\rho J$ are computed via the following formulae:

$$
\rho h=\int_{-\frac{h}{2}}^{\frac{h}{2}} \rho(z) \mathrm{d} z \quad \rho J=\int_{-\frac{h}{2}}^{\frac{h}{2}} \rho(z) z^{2} \mathrm{~d} z
$$

The Власов equations are adopted in this paper, i.e.

$$
f(z)=\frac{3}{2}\left[\frac{z}{h}-\frac{4}{3}\left(\frac{z}{h}\right)^{3}\right] \quad B(z)=0
$$

Then considering the rectangular sandwich plate studied in this paper (refer to Fig. 1), the transverse shear force $Q_{y}$ defined in Eq. (A-7) can be expressed as:

$$
\begin{aligned}
Q_{y}=Q_{4}= & \left\{\left[1-3\left(\frac{h_{2}}{2 h}-\frac{h_{2}^{3}}{6 h^{3}}\right)\right] e_{44}^{1}+3\left(\frac{h_{2}}{2 h}-\frac{h_{2}^{3}}{6 h^{3}}\right) e_{44}^{2}\right\} \psi_{y} \\
& +\left\{\left[1-3\left(\frac{h_{2}}{2 h}-\frac{h_{2}^{3}}{6 h^{3}}\right)\right] e_{45}^{1}+3\left(\frac{h_{2}}{2 h}-\frac{h_{2}^{3}}{6 h^{3}}\right) e_{45}^{2}\right\} \psi_{x}
\end{aligned}
$$

As $e_{45}^{i}=0$ and $e_{44}^{i}=G_{i}=\frac{E_{i}}{2\left(1+\nu_{i}\right)}$, Eq. (A-19) can be simplified as:

$$
Q_{y}=\left\{\left[1-3\left(\frac{h_{2}}{2 h}-\frac{h_{2}^{3}}{6 h^{3}}\right)\right] \frac{E_{1}}{2\left(1+\nu_{1}\right)}+3\left(\frac{h_{2}}{2 h}-\frac{h_{2}^{3}}{6 h^{3}}\right) \frac{E_{2}}{2\left(1+\nu_{2}\right)}\right\} \psi_{y}
$$

by introducing an equivalent shear modulus $G_{z}$ :

$$
G_{z}=\left[1-3\left(\frac{h_{2}}{2 h}-\frac{h_{2}^{3}}{6 h^{3}}\right)\right] \frac{E_{1}}{2\left(1+\nu_{1}\right)}+3\left(\frac{h_{2}}{2 h}-\frac{h_{2}^{3}}{6 h^{3}}\right) \frac{E_{2}}{2\left(1+\nu_{2}\right)}
$$


the transverse shear force $Q_{y}$ will be concisely expressed as:

$$
Q_{y}=G_{z} \psi_{y}
$$

Similarly, $Q_{x}$ will be derived as:

$$
Q_{x}=G_{z} \psi_{x}
$$

The bending and twisting moments defined in Eq. (A-8) can be expressed as:

$$
\begin{aligned}
M_{x}= & \left\{\frac{2 E_{1}}{3\left(1-\nu_{1}^{2}\right)}\left[\left(\frac{h}{2}\right)^{3}-\left(\frac{h_{2}}{2}\right)^{3}\right]+\frac{E_{2}}{3\left(1-\nu_{2}^{2}\right)}\left[\left(\frac{h_{2}}{2}\right)^{3}-\left(-\frac{h_{2}}{2}\right)^{3}\right]\right\} \frac{\partial \beta_{x}}{\partial x} \\
& +\left\{\frac{2 E_{1} \nu_{1}}{3\left(1-\nu_{1}^{2}\right)}\left[\left(\frac{h}{2}\right)^{3}-\left(\frac{h_{2}}{2}\right)^{3}\right]+\frac{E_{2} \nu_{2}}{3\left(1-\nu_{2}^{2}\right)}\left[\left(\frac{h_{2}}{2}\right)^{3}-\left(-\frac{h_{2}}{2}\right)^{3}\right]\right\} \frac{\partial \beta_{y}}{\partial y} \\
M_{y}= & \left\{\frac{2 E_{1} \nu_{1}}{3\left(1-\nu_{1}^{2}\right)}\left[\left(\frac{h}{2}\right)^{3}-\left(\frac{h_{2}}{2}\right)^{3}\right]+\frac{E_{2} \nu_{2}}{3\left(1-\nu_{2}^{2}\right)}\left[\left(\frac{h_{2}}{2}\right)^{3}-\left(-\frac{h_{2}}{2}\right)^{3}\right]\right\} \frac{\partial \beta_{x}}{\partial x} \\
& +\left\{\frac{2 E_{1}}{3\left(1-\nu_{1}^{2}\right)}\left[\left(\frac{h}{2}\right)^{3}-\left(\frac{h_{2}}{2}\right)^{3}\right]+\frac{E_{2}}{3\left(1-\nu_{2}^{2}\right)}\left[\left(\frac{h_{2}}{2}\right)^{3}-\left(-\frac{h_{2}}{2}\right)^{3}\right]\right\} \frac{\partial \beta_{y}}{\partial y} \\
M_{x y}= & \left\{\frac{E_{1}}{3\left(1+\nu_{1}\right)}\left[\left(\frac{h}{2}\right)^{3}-\left(\frac{h_{2}}{2}\right)^{3}\right]+\frac{E_{2}}{6\left(1+\nu_{2}\right)}\left[\left(\frac{h_{2}}{2}\right)^{3}-\left(\frac{-h_{2}}{2}\right)^{3}\right]\right\}\left(\frac{\partial \beta_{x}}{\partial y}+\frac{\partial \beta_{y}}{\partial x}\right)
\end{aligned}
$$

Substituting the expressions of $Q_{x}, Q_{y}, M_{x}, M_{y}$ and $M_{x y}$ into Eq. (A-16), the dynamic equilibrium equations will be obtained as:

$$
\begin{aligned}
& \frac{G_{z} h}{k_{\tau}}\left(\nabla^{2} w+\frac{\partial \beta_{x}}{\partial x}+\frac{\partial \beta_{y}}{\partial y}\right)-\rho h \frac{\partial^{2} w}{\partial t^{2}}+N_{x} \frac{\partial^{2} w}{\partial x^{2}}+N_{y} \frac{\partial^{2} w}{\partial y^{2}}+2 N_{x y} \frac{\partial^{2} w}{\partial x \partial y}+q=0 \\
& D\left(\frac{\partial^{2} \beta_{x}}{\partial x^{2}}+\frac{1-\nu}{2} \frac{\partial^{2} \beta_{x}}{\partial y^{2}}+\frac{1+\nu}{2} \frac{\partial^{2} \beta_{y}}{\partial x \partial y}\right)-\frac{G_{z} h}{k_{\tau}}\left(\beta_{x}+\frac{\partial w}{\partial x}\right)-\rho J \frac{\partial^{2} \beta_{x}}{\partial t^{2}}=0 \\
& D\left(\frac{\partial^{2} \beta_{y}}{\partial y^{2}}+\frac{1-\nu}{2} \frac{\partial^{2} \beta_{y}}{\partial x^{2}}+\frac{1+\nu}{2} \frac{\partial^{2} \beta_{x}}{\partial x \partial y}\right)-\frac{G_{z} h}{k_{\tau}}\left(\beta_{y}+\frac{\partial w}{\partial y}\right)-\rho J \frac{\partial^{2} \beta_{y}}{\partial t^{2}}=0
\end{aligned}
$$

where $D, \nu, \rho h$ and $\rho J$ are defined as the following formulae:

$$
\begin{aligned}
D & =\frac{2 E_{1}}{3\left(1-\nu_{1}^{2}\right)}\left[\left(\frac{h}{2}\right)^{3}-\left(\frac{h_{2}}{2}\right)^{3}\right]+\frac{E_{2}}{3\left(1-\nu_{2}^{2}\right)}\left[\left(\frac{h_{2}}{2}\right)^{3}-\left(-\frac{h_{2}}{2}\right)^{3}\right] \\
\nu & =\frac{1}{3 D}\left\{\frac{2 E_{1} \nu_{1}}{1-\nu_{1}^{2}}\left[\left(\frac{h}{2}\right)^{3}-\left(\frac{h_{2}}{2}\right)^{3}\right]+\frac{E_{2} \nu_{2}}{1-\nu_{2}^{2}}\left[\left(\frac{h_{2}}{2}\right)^{3}-\left(-\frac{h_{2}}{2}\right)^{3}\right]\right\} \\
\rho h & =2 \rho_{1} h_{1}+\rho_{2} h_{2} \\
\rho J & =\frac{2}{3} \rho_{1}\left[\left(\frac{h}{2}\right)^{3}-\left(\frac{h_{2}}{2}\right)^{3}\right]+\frac{1}{3} \rho_{2}\left[\left(\frac{h_{2}}{2}\right)^{3}-\left(-\frac{h_{2}}{2}\right)^{3}\right]
\end{aligned}
$$




\section{References}

[1] C.H. Tu, General equations of sandwich plates under transverse loads and edgewise shears and compressions (in Chinese), Acta Physica Sinica 10 (1954), 395-411.

[2] Q.H. Qin, J.X. Zhang and T.J. Wang, Low velocity impact response of lightweight metal sandwich panel with corrugated core, Materials Research Innovations 15 (2011), 198-200.

[3] F.X. Xin and T.J. Lu, Sound radiation of orthogonally rib-stiffened sandwich structures with cavity absorption, Composites Science and Technology 70 (2010), 2198-2206.

[4] J. Romanoff and P. Varsta, Bending response of web-core sandwich plates, Composite Structures 81 (2007), $292-302$.

[5] C.Y. Tsao, The solution of composite plates by equivalent non-classical theory (in Chinese), Acta Mechanica Solida Sinica 4 (1981), $477-490$.

[6] A. Omrani and I. Tawfiq, Vibro-acoustic analysis of micro-perforated sandwich structure used in space craft industry, Mechanical Systems and Signal Processing 25 (2011), 657-666.

[7] S. Assaf, M. Guerich and P. Cuvelier, Vibration and acoustic response of damped sandwich plates immersed in a light or heavy fluid, Computers and Structures $\mathbf{8 8}$ (2010), 870-878.

[8] S.V. Sorokin, Vibrations of and sound radiation from sandwich plates in heavy fluid loading conditions, Composite Structures $\mathbf{4 8}$ (2000), 219-230.

[9] O. Foin, J. Nicolas and N. Atalla, An efficient tool for predicting the structural acoustic and vibration response of sandwich plates in light or heavy fluid, Applied Acoustics 57 (1999), 213-242.

[10] P. Jeyaraj, N. Ganesan and C. Padmanabhan, Vibration and acoustic response of a composite plate with inherent material damping in a thermal environment, Journal of Sound and Vibration 320 (2009), 322-338.

[11] B.R. Kumar, N. Ganesan and R. Sethuraman, Vibro-acoustic analysis of composite circular disc with various orthotropic properties under thermal environment, International Journal of Vehicle Noise and Vibration 4 (2008), 35-69.

[12] X.W. Yang, Y.M. Li and Q. Geng, Broadband vibro-acoustic response of aircraft in high temperature environment based on hybrid FE-SEA (in Chinese), Acta Aeronautica et Astronautica Sinica 32 (2011), 1851-1859.

[13] Q. Geng, Y.M. Li and X.W. Yang, Vibro-acoustic numerical analysis of thermally stressed aircraft structure (in Chinese), Chinese Journal of Computational Mechanics 29 (2012), 99-104.

[14] Q. Geng and Y.M. Li, Analysis of dynamic and acoustic radiation characters for a flat plate under thermal environments, International Journal of Applied Mechanics 4 (2012), 1250028 (16 pages).

[15] R.B. Hetnarski and M.R. Eslami, Thermal stresses-advanced theory and applications, Springer, Berlin, 2009.

[16] C.C. Sung and J.T. Jan, The response of and sound power radiated by a clamped rectangular plate, Journal of Sound and Vibration 207 (1997), 301-317.

[17] A. Putra and D.J. Thompson, Sound radiation from rectangular baffled and unbaffled plates, Applied Acoustics 71 (2010), $1113-1125$. 

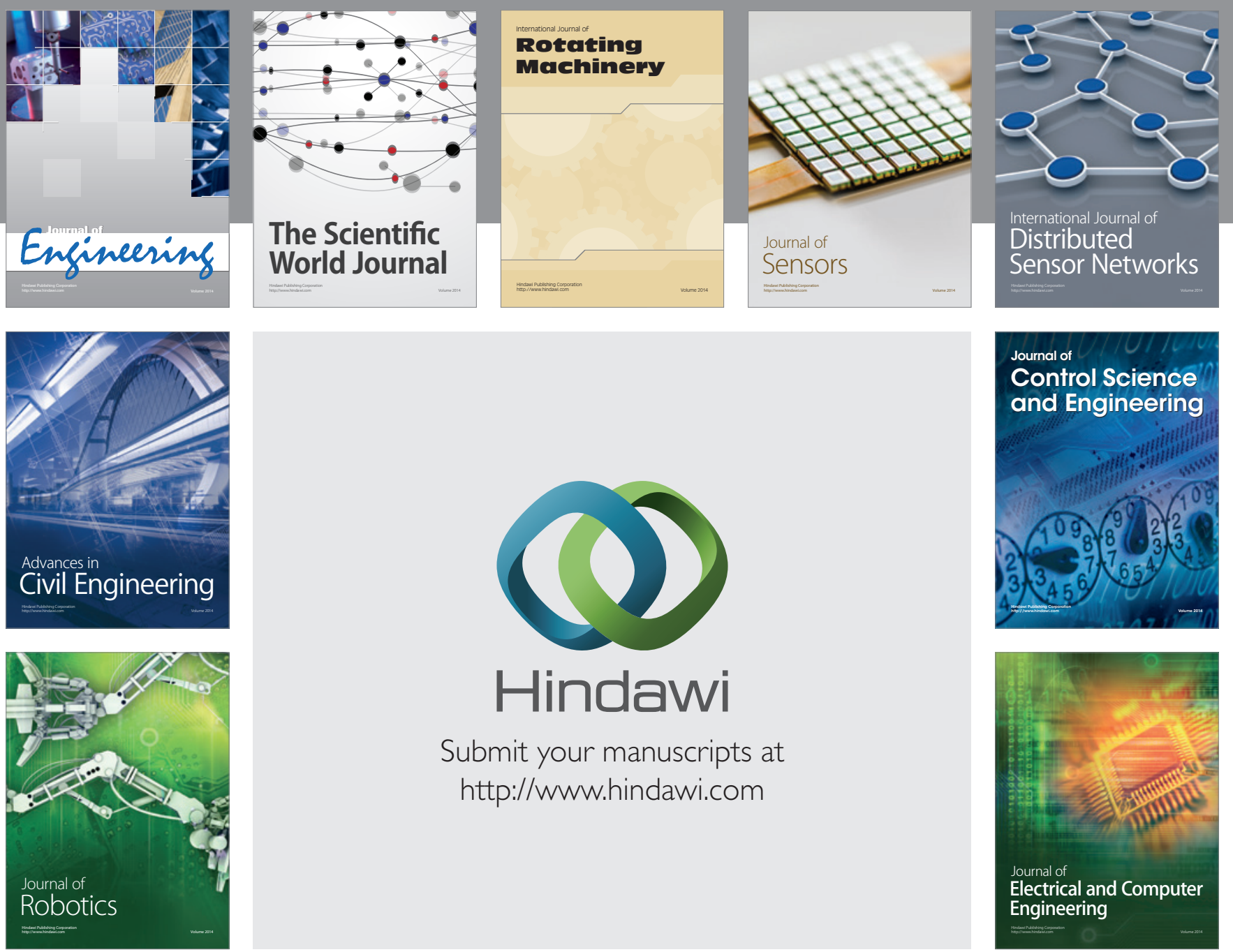

Submit your manuscripts at

http://www.hindawi.com
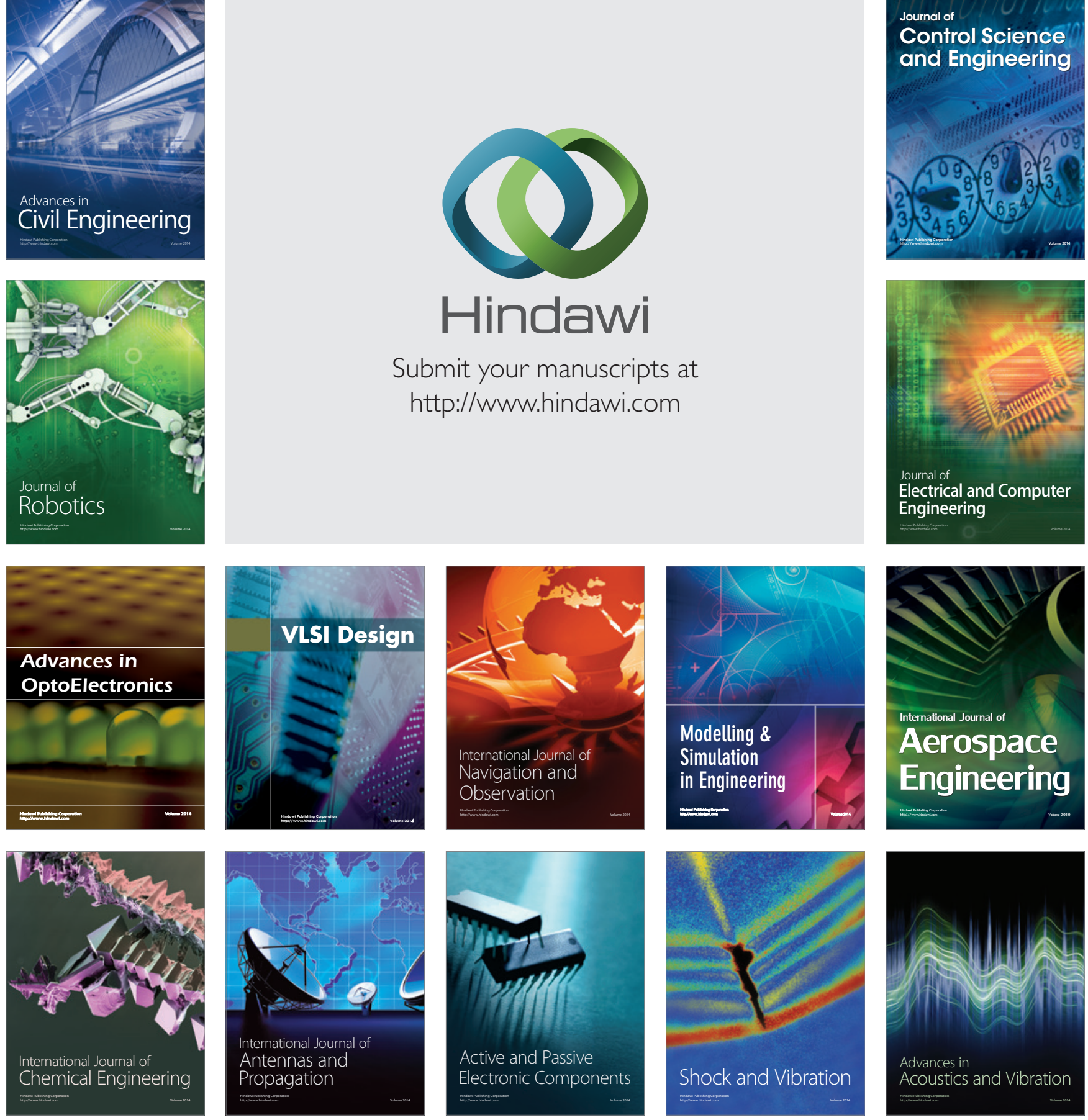